\title{
On a spectral sequence for the cohomology of infinite loop spaces
}

\author{
RUNE HAUGSENG \\ HAYNES MiLleR
}

\begin{abstract}
We study the mod-2 cohomology spectral sequence arising from delooping the Bousfield-Kan cosimplicial space giving the 2-nilpotent completion of a connective spectrum $X$. Under good conditions its $E_{2}$-term is computable as certain nonabelian derived functors evaluated at $\mathrm{H}^{*}(X)$ as a module over the Steenrod algebra, and it converges to the cohomology of $\Omega^{\infty} X$. We provide general methods for computing the $E_{2}$-term, including the construction of a multiplicative spectral sequence of Serre type for cofibration sequences of simplicial commutative algebras. Some simple examples are also considered; in particular, we show that the spectral sequence collapses at $E_{2}$ when $X$ is a suspension spectrum.
\end{abstract}

$18 \mathrm{G} 40,55 \mathrm{P} 47$

\section{Introduction}

This paper explores the relationship between the $\mathbb{F}_{2}$-cohomology $\mathrm{H}^{*} E=\mathrm{H}^{*}\left(E ; \mathbb{F}_{2}\right)$ of a connective spectrum $E$ and that of its associated infinite loop space $\Omega^{\infty} E$.

The starting point is the stabilization map $\mathrm{H}^{*}(E) \rightarrow \mathrm{H}^{*}\left(\Omega^{\infty} E\right)$, induced by the adjunction counit $\Sigma^{\infty} \Omega^{\infty} E \rightarrow E$. This factors through the maximal unstable quotient $D \mathrm{H}^{*}(E)$ of the $A$-module $\mathrm{H}^{*}(E)$ (where $A$ is the Steenrod algebra), and this map then extends over the free unstable algebra $U D H^{*}(E)$. This construction provides the best approximation to $\mathrm{H}^{*}\left(\Omega^{\infty} E\right)$ functorial in the $A$-module $\mathrm{H}^{*}(E)$.

We study a spectral sequence that converges (for $E$ connected and of finite type) to $\mathrm{H}^{*}\left(\Omega^{\infty} E\right)$ and has $E_{2}$-term given by the nonabelian derived functors of $U D$ applied to $\mathrm{H}^{*}(X)$. This is the cohomology spectral sequence associated to the cosimplicial space obtained by applying $\Omega^{\infty}$ to a cosimplicial Adams (or Bousfield-Kan) resolution of the spectrum $E$.

This construction is analogous and in a sense dual to that of [21], where Miller constructed a spectral sequence that converges to $\mathrm{H}_{*}(E)$ by forming a simplicial resolution of $E$ by suspension spectra and applying the zero-space functor $\Omega^{\infty}$. The 
best approximation to the homology of $E$ functorial in the homology of the infinite loop space $\Omega^{\infty} E$ is given by the indecomposables of $\mathrm{H}_{*}\left(\Omega^{\infty} E\right)$ with respect to the Dyer-Lashof operations and products, which are annihilated by the natural map $\mathrm{H}_{*}\left(\Omega^{\infty} E\right) \rightarrow \mathrm{H}_{*}(E)$, and the $E^{2}$-term of the spectral sequence is given by the nonabelian left derived functors of these indecomposables applied to $\mathrm{H}_{*}\left(\Omega^{\infty} E\right)$.

The spectral sequence we study here is hardly new, and has been previously considered (in unpublished work) by Bill Dwyer, Paul Goerss, and no doubt others. Our main contribution here is related to the computation of the $E_{2}$-term, which is of the form $\pi_{*}\left(U V_{\bullet}\right)$, where $V_{\bullet}$ is a simplicial unstable $A$-module. We show that this is determined by a natural short exact sequence of graded unstable modules over the Steenrod algebra, in which the end terms are explicitly given in terms of the graded $A$-module $\pi_{*}\left(V_{\bullet}\right)$. This yields an explicit but mildly nonfunctorial description of the $E_{2}$-term.

This reduces the analysis of the $E_{2}$-term of the spectral sequence to the computation of the derived functors $\mathbb{L}_{*} D$. These derived functors of destabilization have been studied by many authors, including Singer [30; 31], Lannes and Zarati [19], Goerss [11], Kuhn and McCarty [18], and Powell [24].

As an outcome of our computation, we find that the spectral sequence must collapse when $X$ is a connected suspension spectrum, $X=\Sigma^{\infty} B$ for $B$ a connected space. While the spectral sequence collapses by construction when $X$ is a mod-2 EilenbergMac Lane spectrum, its collapse for suspension spectra is a bit of a surprise. This does not yet constitute an independent calculation of the cohomology of $\Omega^{\infty} \Sigma^{\infty} B$, however, since to prove that the spectral sequence collapses we simply compare the size of the $E_{2}$-term with that of the known homology of $\Omega^{\infty} \Sigma^{\infty} B$. It is possible that the collapse follows from Dwyer's description [9] of the behavior of differentials in a spectral sequence of this type.

It would be interesting to compare the spectral sequence we study to that arising from the Goodwillie-Taylor tower of the functor $\Sigma^{\infty} \Omega^{\infty}$, as studied by Kuhn and McCarty [18]. Those authors also relate their spectral sequence to derived functors of destabilization, though in a less direct way than they occur in our spectral sequence; we would like to better understand the relationship between these constructions, which seems analogous to the relationship between the Bousfield-Kan unstable Adams spectral sequence and the spectral sequence arising from the lower central series.

\subsection{Overview}

We construct the spectral sequence in Section 2, and review some background material on simplicial commutative $\mathbb{F}_{2}$-algebras in Section 3. Then in Section 4 we 
compute $\pi_{*} U(M)$ in terms of $\pi_{*} M$, where $M$ is any simplicial $A$-module. We end by discussing some simple examples of the spectral sequence in Section 5.

Acknowledgements Haugseng thanks Vigleik Angeltveit, Paul Goerss and Justin Noel for helpful conversations about this project. Haugseng also thanks the AmericanScandinavian Foundation and the Norway-America Association for financial support during the time much of this work was carried out.

Miller is grateful to the members of the Centre for Symmetry and Deformation at the University of Copenhagen for their hospitality in May 2011, when the initial stages of this work were carried out. The project was jump-started by conversations with Nick Kuhn at a BIRS workshop, and benefited from guidance from Bill Dwyer along the way. This research was carried out in part under NSF grant 0905950.

\section{Definition and convergence of the spectral sequence}

In this section we define the spectral sequence we are interested in, observe that its $E_{2}$-term is described by certain derived functors, and show that it converges under suitable finiteness and connectivity assumptions. More precisely, our goal is to prove the following:

Theorem 2.1 Suppose $X$ is a connected spectrum of finite type, ie $\pi_{*} X$ is 0 for $* \leq 0$ and is a finitely generated abelian group for $*>0$. Then there is a convergent spectral sequence

$$
E_{2}^{s, t}=\mathbb{L}_{-s}(U D)\left(\mathrm{H}^{*} X\right)^{t} \Rightarrow \mathrm{H}^{t+s}\left(\Omega^{\infty} X\right)
$$

Here $\mathbb{L}_{*}(U D)$ denotes the nonabelian derived functors of $U D$, which can be defined as $\pi_{*} U D\left(M_{\bullet}\right)$ where $M_{\bullet}$ is the simplicial free resolution of the $A$-modules $\mathrm{H}^{*} X$.

To define the spectral sequence, recall that for any spectrum $X$ the Eilenberg-Mac Lane ring spectrum $\mathrm{HF}_{2}$ gives a cosimplicial spectrum

$$
P^{\bullet}:=X \wedge \mathrm{HF}_{2}^{\wedge(\bullet+1)}
$$

The homotopy limit of $P^{\bullet}$ is the 2-nilpotent completion $X_{2}^{\wedge}$ of $X$. Since the functor $\Omega^{\infty}$ preserves homotopy limits, the cosimplicial space $\Omega^{\infty} P^{\bullet}$ has homotopy limit $\Omega^{\infty}\left(X_{2}^{\wedge}\right)$. This gives a spectral sequence in cohomology,

$$
E_{2}^{s, t}=\pi_{-s} \mathrm{H}^{t}\left(\Omega^{\infty} P^{\bullet}\right) \Rightarrow \mathrm{H}^{t+s}\left(\Omega^{\infty}\left(X_{2}^{\wedge}\right)\right)
$$

Proposition 2.2 (Bousfield) If $X$ is a connected spectrum of finite type (ie all its homotopy groups are finitely generated), then this spectral sequence converges. 
Proof This follows from (the dual of) the convergence result of [3, Section 4.5].

Lemma 2.3 Suppose $X$ is a connected spectrum of finite type. Then the map $\Omega^{\infty} X \rightarrow \Omega^{\infty}\left(X_{2}^{\wedge}\right)$ exhibits $\Omega^{\infty}\left(X_{2}^{\wedge}\right)$ as the $\mathrm{HF}_{2}$-localization of $\Omega^{\infty} X$. In particular, it induces an equivalence in $\mathrm{HF}_{2}$-cohomology.

Proof If $X$ is connected, then by [2, Theorem 6.6] the 2-nilpotent completion $X_{2}^{\wedge}$ is equivalent to the $\mathrm{HF}_{2}$-localization of $X$; in particular the natural map $X \rightarrow X_{2}^{\wedge}$ induces an equivalence in $\mathrm{HF}_{2}$-cohomology.

Moreover, under the stated assumptions on $X$ the map $X \rightarrow X_{2}^{\wedge}$ induces an isomorphism $\left(\pi_{*} X\right) \otimes \mathbb{Z}_{2}^{\wedge} \stackrel{\sim}{\rightarrow} \pi_{*} X_{2}^{\wedge}$, by [2, Proposition 2.5]. Since $X$ is connected, the space $\Omega^{\infty} X$ is nilpotent, and so by [4, Example VI.5.2] the map $\Omega^{\infty} X \rightarrow\left(\Omega^{\infty} X\right) \hat{2}^{\wedge}$ also induces an isomorphism $\left(\pi_{*} \Omega^{\infty} X\right) \otimes \mathbb{Z}_{2}^{\wedge} \sim \pi_{*}\left(\Omega^{\infty} X\right)_{2}^{\wedge}$. Since $\Omega^{\infty}\left(X_{2}^{\wedge}\right)$ is 2-complete, the map $\Omega^{\infty} X \rightarrow \Omega^{\infty}\left(X_{2}^{\wedge}\right)$ factors through $\left(\Omega^{\infty} X\right)_{2}^{\wedge}$; we know that two of the maps in the resulting commutative diagram

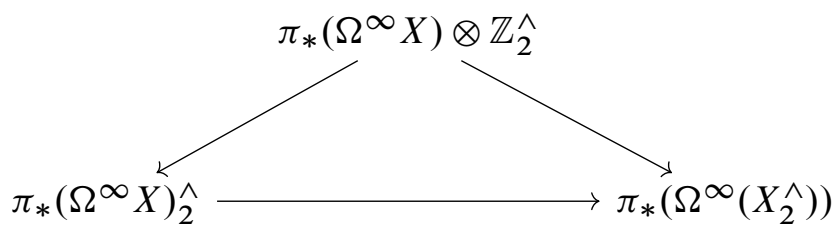

are isomorphisms, hence the map $\left(\Omega^{\infty} X\right) \wedge \rightarrow \Omega^{\infty}\left(X_{2}^{\wedge}\right)$ is a weak equivalence. The result follows since under our assumptions the map $\Omega^{\infty} X \rightarrow\left(\Omega^{\infty} X\right) \hat{2}_{2}^{\wedge}$ exhibits $\left(\Omega^{\infty} X\right) \hat{2}^{\wedge}$ as the $\mathrm{HF}_{2}$-localization of $\Omega^{\infty} X$ by [4, Proposition VI.5.3].

Under these finiteness assumptions the spectral sequence thus converges to the mod-2 cohomology of $\Omega^{\infty} X$. To describe the $E_{2}$-term more algebraically, we appeal to Serre's computation of the cohomology of Eilenberg-Mac Lane spaces. To state this we must first recall some definitions:

Definition 2.4 Let $\operatorname{Mod}_{A}$ be the category of (graded) $A$-modules, and let $\mathcal{U}$ be the full subcategory of unstable modules, ie $A$-modules $M$ such that if $x \in M_{n}$ then $\operatorname{Sq}^{i} x=0$ for $i>n$. We define $D: \operatorname{Mod}_{A} \rightarrow \mathcal{U}$ to be the destabilization functor, which sends an $A$-module $M$ to its quotient by the submodule generated by $\mathrm{Sq}^{i} x$ where $x \in M_{n}$ and $i>n$; the functor $D$ is left adjoint to the inclusion $\mathcal{U} \hookrightarrow \operatorname{Mod}_{A}$.

Definition 2.5 Let $\mathcal{K}$ be the category of unstable algebras over the Steenrod algebra $A$, ie augmented commutative $A$-algebras $R$ that are unstable as $A$-modules, 
with $x^{2}=\operatorname{Sq}^{n} x$ for all $x \in R_{n}$. We define $U: \mathcal{U} \rightarrow \mathcal{K}$ to be the free unstable algebra functor, which sends $M \in \mathcal{U}$ to

$$
S(M) /\left(x^{2}-\mathrm{Sq}^{|x|} x\right),
$$

where $S$ is the free graded symmetric algebra functor; this functor is left adjoint to the forgetful functor $\mathcal{K} \rightarrow \mathcal{U}$.

Theorem 2.6 (Serre [29]) If $M$ is an Eilenberg-Mac Lane spectrum of finite type, then the natural map $\mathrm{H}^{*}(M) \rightarrow \mathrm{H}^{*}\left(\Omega^{\infty} M\right)$ induces an isomorphism

$$
U D\left(\mathrm{H}^{*} M\right) \stackrel{\sim}{\longrightarrow} \mathrm{H}^{*}\left(\Omega^{\infty} M\right) .
$$

For any $n>0$ the spectrum $X \wedge \mathrm{HF}_{2}^{\wedge n}$ is a wedge of suspensions of EilenbergMac Lane spectra, so this theorem allows us to rewrite the $E_{2}$-term of our spectral sequence as

$$
E_{2}^{s, t}=\pi_{-s} U D\left(\mathrm{H}^{*} P^{\bullet}\right)^{t}
$$

But by the Künneth theorem $\mathrm{H}^{*}\left(P^{n}\right)$ is isomorphic to $\mathrm{H}^{*}(X) \otimes A^{\otimes n+1}$, and in fact the simplicial $A$-module $\mathrm{H}^{*}\left(P^{\bullet}\right)$ is the standard cotriple resolution of $\mathrm{H}^{*} X$. The $A$-modules $\pi_{*} U D\left(\mathrm{H}^{*} P^{\bullet}\right)$ can therefore be interpreted as the (nonabelian) derived functors $\mathbb{L}_{*}(U D)$ of $U D$ evaluated at $\mathrm{H}^{*} X$. This completes the proof of Theorem 2.1.

Remark 2.7 Our spectral sequence is of the type considered by Dwyer in [9], so by [9, Proposition 2.3] it is a spectral sequence of $A$-algebras. By (the dual of) results of Hackney [15] it is actually a spectral sequence of Hopf algebras.

\section{Simplicial commutative $\mathbb{F}_{2}$-algebras}

In this section we first review some background material on simplicial commutative (graded) algebras: we recall the model category structure on simplicial commutative algebras in Section 3.1 and in Section 3.2 we review the higher divided square operations in the homotopy groups of simplicial commutative algebras. Then in Section 3.3 we discuss filtered algebras and modules from an abstract point of view, and finally in Section 3.4 we use this material to construct a "Serre spectral sequence" for cofiber sequences of simplicial commutative algebras. 


\subsection{Model category structure}

We will make use of a model category structure on simplicial augmented commutative graded $\mathbb{F}_{2}$-algebras. This is an instance of a general class of model categories constructed by Quillen [25], and is also described by Miller [22]:

Theorem 3.1 There is a simplicial model category structure on the category of simplicial augmented graded commutative $\mathbb{F}_{2}$-algebras where a morphism is a weak equivalence or fibration if the underlying map of simplicial sets is a weak equivalence or Kan fibration.

Remark 3.2 For us graded will mean $\mathbb{N}$-graded rather than $\mathbb{Z}$-graded. To avoid confusion, let us also mention that we do not require that a graded $\mathbb{F}_{2}$-algebra $A$ has $A_{0}=\mathbb{F}_{2}$, as is sometimes assumed in the literature.

Remark 3.3 Since a simplicial graded commutative $\mathbb{F}_{2}$-algebra is a simplicial group, a morphism $f: A \rightarrow B$ is a fibration if and only if the induced map $A \rightarrow B \times_{\pi_{0} B} \pi_{0} A$ is surjective. In particular, every object is fibrant.

Theorem 3.4 (Rezk) This model structure on simplicial augmented graded commutative $\mathbb{F}_{2}$-algebras is proper.

Proof This follows from the properness criterion of [26, Theorem 9.1], since polynomial algebras are flat and thus tensoring with them preserves weak equivalences.

We now recall Miller's description of the cofibrations in this model category:

Definition 3.5 A morphism $f: A \rightarrow B$ of simplicial augmented commutative $\mathbb{F}_{2}-$ algebras is almost-free if for every $n \geq 0$ there is a subspace $V_{n}$ of the augmentation ideal $I B_{n}$ and maps

$$
\begin{array}{ll}
\delta_{i}: V_{n} \rightarrow V_{n-1} & \text { for } 1 \leq i \leq n, \\
\sigma_{i}: V_{n} \rightarrow V_{n+1} & \text { for } 0 \leq i \leq n,
\end{array}
$$

so that the induced map $A_{n} \otimes S\left(V_{n}\right) \rightarrow B_{n}$ is an isomorphism for all $n$ and the following diagrams commute:

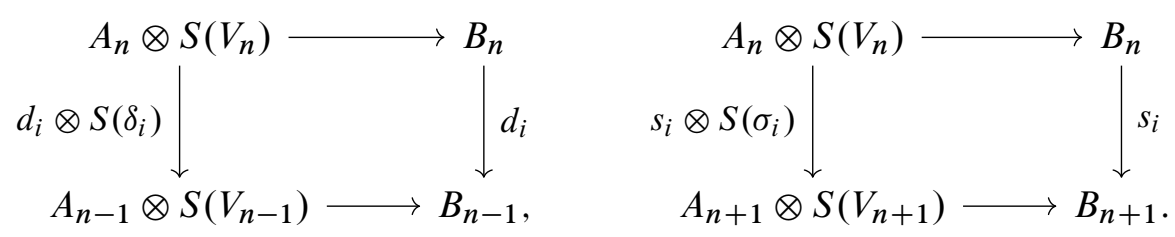


In other words, all the face and degeneracy maps except $d_{0}$ are induced from maps between the $V_{n}$.

Remark 3.6 The definition of almost free morphisms in [22] is wrong and was corrected in [23].

Theorem 3.7 (Miller, [22, Corollary 3.5]) A morphism of simplicial augmented commutative $\mathbb{F}_{2}$-algebras is a cofibration if and only if it is a retract of an almost-free morphism.

\subsection{Higher divided square operations}

In this subsection we review the higher divided square operations on the homotopy groups of simplicial commutative $\mathbb{F}_{2}$-algebras. These operations were initially introduced by Cartan [5], and have subsequently also been studied by Bousfield [1] and Dwyer [10].

Definition 3.8 If $V$ is a simplicial $\mathbb{F}_{2}$-vector space, we write $C(V)$ for the unnormalized chain complex of $V$ (obtained by taking the alternating sum of the face maps as the differential) and $N(V)$ for the normalized chain complex, given by

$$
N_{k} X=\bigcap_{i \neq 0} \operatorname{ker}\left(d_{i}: X_{k} \rightarrow X_{k-1}\right)
$$

with differential $d_{0}$. These chain complexes are quasi-isomorphic, and their homology groups are the same as the homotopy groups of $V$, regarded as a simplicial set.

Theorem 3.9 (Dwyer [10]) Let $A$ be a simplicial commutative $\mathbb{F}_{2}$-algebra.

(i) There are maps $\delta_{i}: C(A)_{n} \rightarrow N(A)_{n+i}$ for $i \geq 1$ that satisfy

$$
d \delta_{i}(a)=\left\{\begin{array}{cl}
\delta_{i}(d a) & \text { if } n>i>1, \\
\delta_{1}(d a)+\phi(a) & \text { if } i=1 \text { and } n>1, \\
a d a & \text { if } i=n>1, \\
a d a+\phi(a) & \text { if } n=i=1 .
\end{array}\right.
$$

Here $\phi(a)$ denotes the image in $N(A)$ of the square $a^{2}$ of $a$ in the multiplication on $A_{n}$.

(ii) In particular, there are higher divided square operations $\delta_{i}: \pi_{n}(A) \rightarrow \pi_{n+i}(A)$ for $2 \leq i \leq n$. If $a^{2}=0$ for all $a \in A$, then there is also an operation $\delta_{1}: \pi_{n}(A) \rightarrow \pi_{n+1}(A)$ for $n \geq 1$.

(iii) These operations have the following properties: 
(1) $\delta_{i}: \pi_{n} A \rightarrow \pi_{n+i} A$ is an additive homomorphism for $2 \leq i<n$, and $\delta_{n}$ satisfies

$$
\delta_{n}(x+y)=\delta_{n}(x)+\delta_{n}(y)+x y .
$$

(2) $\delta_{i}$ acts on products as follows:

$$
\delta_{i}(x y)=\left\{\begin{array}{cl}
x^{2} \delta_{i}(y) & \text { if } x \in \pi_{0} A \\
y^{2} \delta_{i}(x) & \text { if } y \in \pi_{0} A \\
0 & \text { otherwise. }
\end{array}\right.
$$

(3) ("Adém relations") If $i<2 j$ then

$$
\delta_{i} \delta_{j}(x)=\sum_{(i+1) / 2 \leq s \leq(i+j) / 3}\left(\begin{array}{c}
j-i+s-1 \\
j-s
\end{array}\right) \delta_{i+j-s} \delta_{s}(x) .
$$

Remark 3.10 Part (i) is not quite true using Dwyer's definition of the chain-level operations in [10], but is correct for the variant due to Goerss [12].

Remark 3.11 The upper bound in the "Adém relation" above differs from that in [10], which does not give a sum of admissible operations; this form of the relation was proved by Goerss and Lada [13] and implies, by the same proof as for Steenrod operations, that composites of $\delta$-operations are spanned by admissible composites:

Definition 3.12 A sequence $I=\left(i_{1}, \ldots, i_{k}\right)$ is admissible if $i_{t} \geq 2 i_{t+1}$ for all $t$. A composite $\delta_{I}:=\delta_{i_{1}} \delta_{i_{2}} \cdots \delta_{i_{k}}$ is admissible if $I$ is.

Corollary 3.13 Any composite of $\delta$-operations can be written as a sum of admissible ones.

Remark 3.14 For any $x$ in $\pi_{*} A$ in positive degree we have $x^{2}=0$. Therefore Theorem 3.9(iii)(1)-(2) imply that the top operation $\delta_{n}$ on $\pi_{n}$ is a divided square, whence the name "higher divided squares" for the $\delta_{i}$-operations.

Dwyer proves Theorem 3.9 by computing the homotopy groups in the universal case, namely the symmetric algebra $s(V)$ on a simplicial vector space $V$. We will now recall the result of this computation, as well as the analogous result for exterior algebras (both of which are originally due to Bousfield [1]). To state this we make use of the following theorem of Dold:

Theorem 3.15 (Dold [7, 5.17]) Let Vect be the category of $\mathbb{F}_{2}$-vector spaces, and grVect that of graded $\mathbb{F}_{2}$-vector spaces. For any functor $F$ : Vect ${ }^{\times n} \rightarrow$ Vect there 
exists a functor $\mathfrak{F}:$ grVect $^{\times n} \rightarrow$ grVect such that for $V_{1}, \ldots, V_{n}$ simplicial $\mathbb{F}_{2}$-vector spaces there is a natural isomorphism

$$
\pi_{*} F\left(V_{1}, \ldots, V_{n}\right) \cong \mathfrak{F}\left(\pi_{*} V_{1}, \ldots, \pi_{*} V_{n}\right),
$$

where on the left-hand side we take the homotopy of the diagonal of the multisimplicial $\mathbb{F}_{2}$-vector space $F\left(V_{1}, \ldots, V_{n}\right)$.

Example 3.16 The Eilenberg-Zilber theorem implies that if $F$ is the tensor product functor, then $\mathfrak{F}$ is the graded tensor product of graded vector spaces.

In the symmetric algebra case, the functor $\mathfrak{s}$ such that $\pi_{*} S(V)=\mathfrak{s}\left(\pi_{*} V\right)$ has the following description:

Theorem 3.17 (Bousfield [1], Dwyer [10]) The functor $\mathfrak{s}$ sends a graded vector space $V$ to that freely generated on $V$ by a commutative product and operations $\delta_{i}$ satisfying the relations stated in Theorem 3.9 above as well as the relation $x^{2}=0$ for all $x$ of positive degree.

If $B$ is a graded basis for $V$, then $\mathfrak{s}\left(\pi_{*}(V)\right)$ is the free commutative algebra (modulo the relation $x^{2}=0$ for $\left.|x|>0\right)$ generated by elements $\delta_{I} v$ in degree $|v|+i_{1}+\cdots+i_{k}$ for admissible sequences $I=\left(i_{1}, \ldots, i_{k}\right)$ with $i_{k} \geq 2$ of excess $e(I):=i_{1}-i_{2}-\cdots-i_{k}$ at most $|v|$, as $v$ runs over $B$.

Let $s_{k}(V)$ be the subspace of the symmetric algebra $s(V)$ spanned by products of length $k$; it also is a functor Vect $\rightarrow$ Vect. Implicit in Theorem 3.17 is the following description of the functor $\mathfrak{s}_{k}$ such that $\pi_{*} s_{k}(V)=\mathfrak{s}_{k}\left(\pi_{*} V\right)$.

Theorem 3.18 Suppose $V$ is a graded vector space. Define inductively a weight function on products $\delta_{I_{1}}\left(v_{1}\right) \cdots \delta_{I_{n}}\left(v_{n}\right)$ where $v_{i} \in V$ and the $I_{i}$ are admissible sequences by

$$
\begin{aligned}
\operatorname{wt}(v) & =1 \quad \text { for } v \text { in } V, \\
\operatorname{wt}(x y) & =\operatorname{wt}(x)+\operatorname{wt}(y), \\
\operatorname{wt}\left(\delta_{i}(x)\right) & =2 \operatorname{wt}(x) .
\end{aligned}
$$

Then $\mathfrak{s}_{k}(V)$ is the subspace of $\mathfrak{s}(V)$ spanned by elements of weight $k$.

Let $e(V)$ denote the exterior algebra on $V$ and $e_{k}(V)$ its subspace of products of length $k$. Then there are functors $\mathfrak{e}$ and $\mathfrak{e}_{k}$ such that $\pi_{*} e(V)=\mathfrak{e}\left(\pi_{*} V\right)$ and $\pi_{*} e_{k}(V)=\mathfrak{e}_{k}\left(\pi_{*} V\right)$ for a simplicial vector space $V$. These were also computed by Bousfield: 
Theorem 3.19 (Bousfield [1]) The functor $\mathfrak{e}$ sends a graded vector space $V$ to that freely generated on $V$ by a commutative product and operations $\delta_{i}$ (now with $i=1$ allowed) satisfying the same relations as in the symmetric case, and with $x^{2}=0$ for all $x$. Thus $\mathfrak{e}(V)$ is generated by $v \in V$ and symbols $\delta_{I} v$ for admissible sequences $I=\left(i_{1}, \ldots, i_{k}\right)$ (now with $i_{k} \geq 1$ ) of excess $\leq|v|$; the element $\delta_{I} v$ is again in degree $|v|+i_{1}+\cdots+i_{k}$. Defining the weight of such a generator as before, the graded vector space $\mathfrak{e}_{k} V$ is the subspace of $\mathfrak{e} V$ spanned by elements of weight $k$.

Remark 3.20 The same results hold in the graded case. We will use capital letters for the graded versions of the functors considered above: so $S(V)$ denotes the free graded symmetric algebra on the graded vector space $V$, etc. The higher divided power operations $\delta_{i}$ double the internal degree.

\subsection{An abstract approach to filtered algebras and modules}

Given filtered algebras $A, B$ and $C$, and maps $A \rightarrow B$ and $A \rightarrow C$, we would like to construct a filtration on the relative tensor product $B \otimes_{A} C$ whose associated graded is the relative tensor product $E^{0} B \otimes_{E^{0} A} E^{0} C$ of graded algebras, where $E^{0} A$ denotes the associated graded algebra of the filtered algebra $A$. Our goal in this section is to show that this is possible, provided we allow ourselves to take cofibrant replacements of these algebras in a suitable model category. We will do this by considering filtered objects, and in particular filtered modules over a filtered algebra, from an abstract perspective.

Let $\mathbf{N}$ denote the partially ordered set of natural numbers $0,1, \ldots$, considered as a category. If $\mathbf{C}$ is a category, we write $\operatorname{Seq}(\mathbf{C})$ for the category $\operatorname{Fun}(\mathbf{N}, \mathbf{C})$ of sequences of morphisms in $\mathbf{C}$. A filtered object of $\mathbf{C}$, if $\mathbf{C}$ is for example the category of chain complexes of abelian groups, can then be thought of as a certain kind of object of $\operatorname{Seq}(\mathbf{C})$.

Addition of natural numbers is a symmetric monoidal structure on $\mathbf{N}$, so if $\mathbf{C}$ is a category with finite colimits and a symmetric monoidal structure that commutes with finite colimits in each variable (for short, $\mathbf{C}$ is a symmetric monoidal category compatible with finite colimits) we can equip $\operatorname{Seq}(\mathbf{C})$ with the Day convolution tensor product. This has as unit the constant sequence

$$
I \rightarrow I \rightarrow \cdots
$$

with value the unit $I$ in $\mathbf{C}$, and if $A$ and $B$ are sequences in $\mathbf{C}$ their tensor product $A \otimes B$ is given by

$$
(A \otimes B)_{n}=\operatorname{colim}_{i+j \leq n} A_{i} \otimes B_{j}
$$


Remark 3.21 A simple cofinality argument shows that this colimit is isomorphic to the iterated pushout

$$
A_{n} \otimes B_{0} \amalg_{A_{n-1} \otimes B_{0}} A_{n-1} \otimes B_{1} \amalg_{A_{n-2} \otimes B_{1}} \cdots \amalg_{A_{0} \otimes B_{n-1}} A_{0} \otimes B_{n},
$$

which we can also describe as the coequalizer of the two obvious maps

$$
\coprod_{s+t=n-1} A_{s} \otimes B_{t} \rightrightarrows \coprod_{i+j=n} A_{i} \otimes B_{j}
$$

In other words, $(A \otimes B)_{n}$ is the quotient of $\bigsqcup_{i+j=n} A_{i} \otimes B_{j}$ where we identify the images of $A_{s} \otimes B_{t}$ with $s+t=n-1$ in $A_{s+1} \otimes B_{t}$ and $A_{s} \otimes B_{t+1}$.

If $A$ is an algebra object in $\operatorname{Seq}(\mathbf{C})$, the Day convolution on $\operatorname{Seq}(\mathbf{C})$ induces a relative tensor product on the category $\operatorname{Mod}_{A}(\operatorname{Seq}(\mathbf{C}))$ of $A$-modules, given by the (reflexive) coequalizer

$$
M \otimes A \otimes N \rightrightarrows M \otimes N \rightarrow M \otimes_{A} N
$$

where $M$ and $N$ are $A$-modules in $\operatorname{Seq}(\mathbf{C})$. If $\mathbf{C}$ is, for instance, chain complexes, then a filtered algebra $A$ in $\mathbf{C}$ is in particular an algebra object of $\operatorname{Seq}(\mathbf{C})$, and filtered $A$-modules $M$ and $N$ are also modules for $A$ in $\operatorname{Seq}(\mathbf{C})$. The tensor product of $A$-modules then yields an object $M \otimes_{A} N$ in $\operatorname{Seq}(\mathbf{C})$, but in general this need not be a filtered object of $\mathbf{C}$, as the maps in this sequence need no longer be monomorphisms. However, we can use a model structure on $\mathbf{C}$ to deal with this: If $\mathbf{C}$ is a combinatorial model category, we can equip $\operatorname{Seq}(\mathbf{C})$ with the projective model structure. A cofibrant object in $\operatorname{Seq}(\mathbf{C})$ is then a sequence

$$
A_{0} \rightarrow A_{1} \rightarrow \cdots
$$

where the objects $A_{i}$ are all cofibrant, and the morphisms $A_{i} \rightarrow A_{i+1}$ are all cofibrations. If cofibrations in $\mathbf{C}$ are monomorphisms, as they are for chain complexes or simplicial algebras, then a cofibrant object of $\operatorname{Seq}(\mathbf{C})$ is thus in particular a filtered object.

The Day convolution tensor product interacts well with this model structure:

Proposition 3.22 (Isaacson) Let $\mathbf{C}$ be a symmetric monoidal combinatorial model category that satisfies the monoid axiom. Then $\operatorname{Seq}(\mathbf{C})$ is also a symmetric monoidal combinatorial model category with respect to the Day convolution and satisfies the monoid axiom.

Proof This is a special case of [16, Proposition 8.4].

We can now apply results of Schwede and Shipley to get the following: 
Corollary 3.23 Let $\mathbf{C}$ be a symmetric monoidal combinatorial model category that satisfies the monoid axiom, and suppose $A$ is a commutative algebra object in $\operatorname{Seq}(\mathbf{C})$. Then:

(i) The category $\mathrm{Alg}(\mathrm{Seq} \mathbf{C})$ of associative algebra objects of $\mathrm{Seq}(\mathbf{C})$ is a combinatorial model category. The forgetful functor to $\operatorname{Seq}(\mathbf{C})$ creates weak equivalences and fibrations, and the free-forgetful adjunction

$$
\operatorname{Seq}(\mathbf{C}) \rightleftarrows \operatorname{Alg}(\operatorname{Seq}(\mathbf{C}))
$$

is a Quillen adjunction.

(ii) If the unit of $\mathbf{C}$ is cofibrant, then the forgetful functor $\operatorname{Alg}(\operatorname{Seq}(\mathbf{C})) \rightarrow \operatorname{Seq}(\mathbf{C})$ preserves cofibrant object.

(iii) The category $\operatorname{Mod}_{A}(\operatorname{Seq}(\mathbf{C}))$ of $A$-modules is a symmetric monoidal combinatorial model category satisfying the monoid axiom. The forgetful functor to $\operatorname{Seq}(\mathbf{C})$ creates weak equivalences and fibrations, and the free-forgetful adjunction

$$
F_{A}: \operatorname{Seq}(\mathbf{C}) \rightleftarrows \operatorname{Mod}_{A}(\operatorname{Seq}(\mathbf{C})): U_{A}
$$

is a Quillen adjunction.

(iv) If the underlying object of $A$ is cofibrant in $\operatorname{Seq}(\mathbf{C})$ then the forgetful functor $U_{A}$ also preserves cofibrations.

Proof (i), (ii) and (iii) follow from [27, Theorem 4.1], and (iv) is an easy consequence of the construction of the model structure using [27, Lemma 2.3]: If $I$ is a set of generating cofibrations in $\operatorname{Seq}(\mathbf{C})$, then $F_{A}(I)$ is a set of generating cofibrations in $A$-modules. The triple $U_{A} F_{A}$ is $A \otimes-$, which is a left Quillen functor if $A$ is cofibrant in $\operatorname{Seq}(\mathbf{C})$. Thus $U_{A}$ takes the generating cofibrations to cofibrations. But $U_{A}$ also preserves colimits, so as any cofibration is a transfinite composite of pushouts of generating cofibrations this means it preserves all cofibrations.

Corollary 3.24 Let $\mathbf{C}$ be a symmetric monoidal combinatorial model category that satisfies the monoid axiom, and suppose $A$ is a commutative algebra object in $\mathrm{Seq}(\mathbf{C})$ whose underlying object in $\operatorname{Seq}(\mathbf{C})$ is cofibrant. If $M$ and $N$ are cofibrant $A$-modules, then $M \otimes_{A} N$ is a cofibrant object of $\operatorname{Seq}(\mathbf{C})$.

This is the result we need to make our spectral sequence: if $A$ is a suitable filtered algebra in, say, chain complexes, and $M$ and $N$ are filtered $A$-modules, we can take cofibrant replacements for them in the model structure on $\operatorname{Mod}_{A}(\operatorname{Seq}(\mathbf{C}))$ to get a cofibrant relative tensor product over $A$, which is in particular a filtered object and so gives a spectral sequence. 
Next we want to analyze the associated graded object of such a relative tensor product, which will allow us to describe the $E^{1}$-page of our spectral sequence:

Definition 3.25 Let $\mathbf{C}$ be a category with finite colimits and a zero object 0 . Denote the product $\prod_{i=0}^{\infty} \mathbf{C}$ by $\operatorname{Gr}(\mathbf{C})$ and write $\operatorname{Triv}: \operatorname{Gr}(\mathbf{C}) \rightarrow \operatorname{Seq}(\mathbf{C})$ for the functor that sends $\left(X_{i}\right)_{i \in \mathbb{N}}$ to the sequence

$$
X_{0} \stackrel{0}{\rightarrow} X_{1} \stackrel{0}{\rightarrow} \cdots
$$

This has a left adjoint $E^{0}: \operatorname{Seq}(\mathbf{C}) \rightarrow \operatorname{Gr}(\mathbf{C})$, the associated graded functor. We have $\left(E^{0} A\right)_{0}=A_{0}$ and $\left(E^{0} A\right)_{n}$ for $n>0$ is the quotient $A_{n} / A_{n-1}$, ie the pushout

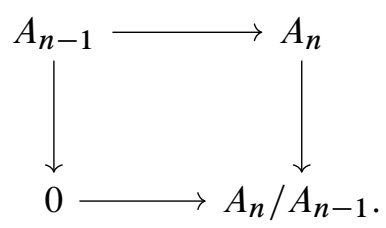

If $\mathbf{C}$ has a symmetric monoidal structure, then we can equip $\operatorname{Gr}(\mathbf{C})$ with a graded tensor product (another Day convolution), given by

$$
(X \otimes Y)_{n}=\coprod_{i+j=n} X_{i} \otimes Y_{j}
$$

The unit is $(I, 0,0, \ldots)$.

Proposition 3.26 Let $\mathbf{C}$ be a symmetric monoidal category compatible with finite colimits that has a zero object. Then the functor $E^{0}: \operatorname{Seq}(\mathbf{C}) \rightarrow \operatorname{Gr}(\mathbf{C})$ is symmetric monoidal.

Proof $E^{0}$ clearly preserves the unit, so it suffices to show that there is a natural isomorphism $E^{0} M \otimes E^{0} N \stackrel{\sim}{\longrightarrow} E^{0}(M \otimes N)$.

By definition, $E_{n}^{0}(M \otimes N)$ is the cofiber of $(M \otimes N)_{n-1} \rightarrow(M \otimes N)_{n}$. For $n \in \mathbf{N}$, let $(\mathbf{N} \times \mathbf{N})_{\leq n}$ denote the full subcategory of $\mathbf{N} \times \mathbf{N}$ spanned by the objects $(i, j)$ with $i+j \leq n$; if $M \otimes N$ denotes the composite functor

$$
\mathbf{N} \times \mathbf{N} \stackrel{M \times N}{\longrightarrow} \mathbf{C} \times \mathbf{C} \stackrel{\otimes}{\longrightarrow} \mathbf{C},
$$

then $(M \otimes N)_{n}$ is by definition given by the colimit of $M \otimes N$ restricted to $(\mathbf{N} \times \mathbf{N})_{\leq n}$. Let $\alpha$ denote the inclusion $(\mathbf{N} \times \mathbf{N})_{\leq(n-1)} \hookrightarrow(\mathbf{N} \times \mathbf{N})_{\leq n}$; then $(M \otimes N)_{n-1}$ is isomorphic to the colimit of the left Kan extension $\left.\alpha_{!}(M \otimes N)\right|_{(\mathbf{N} \times \mathbf{N})_{\leq(n-1)}}$. Thinking of 0 as the constant diagram of shape $(\mathbf{N} \times \mathbf{N})_{\leq n}$ with value 0 , we can write $E_{n}^{0}(M \otimes N)$ 
as the pushout of two maps between colimits of diagrams of the same shape. Moreover, these maps arise from natural transformations, so since colimits commute we can identify $E_{n}^{0}(M \otimes N)$ with the colimit of the functor $\beta:(\mathbf{N} \times \mathbf{N})_{\leq n} \rightarrow \mathbf{C}$ that assigns to $(i, j)$ the cofiber of the map

$$
\phi_{i, j}:\left(\left.\alpha_{!}(M \otimes N)\right|_{(\mathbf{N} \times \mathbf{N})_{\leq(n-1)}}\right)(i, j) \rightarrow(M \otimes N)(i, j) .
$$

If $i+j<n$ then, since $(\mathbf{N} \times \mathbf{N})_{\leq(n-1)}$ is a full subcategory of $(\mathbf{N} \times \mathbf{N})_{\leq n}$, the map $\phi_{i, j}$ is an isomorphism, so $\beta(i, j) \cong 0$. It follows that the colimit of $\beta$ is just the coproduct $\bigsqcup_{i+j=n} \beta(i, j)$, and it remains to show that $\beta(i, j)$ is isomorphic to $E_{i}^{0} M \otimes E_{j}^{0} N$.

If $i+j=n$, let $(\mathbf{N} \times \mathbf{N})_{<(i, j)}$ be the full subcategory of $\mathbf{N} \times \mathbf{N}$ spanned by the objects $(x, y)$ with $x \leq i$ and $y \leq j$, except for $(i, j)$. Then by definition we have that $\alpha_{!}\left(\left.(M \otimes N)\right|_{(\mathbf{N} \times \mathbf{N})_{\leq(n-1)}}\right)(i, j)$ is the colimit of $M \otimes N$ restricted to $(\mathbf{N} \times \mathbf{N})_{<(i, j)}$.

Write $(\mathbf{N} \times \mathbf{N})_{<(i, j)}^{0}$ for the full subcategory

$$
(i-1, j) \leftarrow(i-1, j-1) \rightarrow(i, j-1)
$$

of $(\mathbf{N} \times \mathbf{N})_{<(i, j)}$. We claim the inclusion $(\mathbf{N} \times \mathbf{N})_{<(i, j)}^{0} \hookrightarrow(\mathbf{N} \times \mathbf{N})_{<(i, j)}$ is cofinal, and so gives an isomorphism of colimits. By [20, Theorem IX.3.1], to see this it suffices to show that the categories

$$
\left((\mathbf{N} \times \mathbf{N})_{<(i, j)}^{0}\right)_{(x, y) /}=(\mathbf{N} \times \mathbf{N})_{<(i, j)}^{0} \times(\mathbf{N} \times \mathbf{N})_{<(i, j)}\left((\mathbf{N} \times \mathbf{N})_{<(i, j)}\right)_{(x, y) /}
$$

are nonempty and connected. But this category is either all of $(\mathbf{N} \times \mathbf{N})_{<(i, j)}^{0}$ if $x \leq i-1$ and $y \leq j-1$, or the single object $(i, j-1)$ if $x=i$, or the single object $(i-1, j)$ if $y=j$; these are certainly all nonempty and connected. We may thus identify $\alpha_{!}\left(\left.(M \otimes N)\right|_{(\mathbf{N} \times \mathbf{N})_{\leq(n-1)}}\right)(i, j)$ with the pushout $M_{i} \otimes N_{j-1} \amalg_{M_{i-1} \otimes N_{j-1}} M_{i-1} \otimes N_{j}$ and $\beta(i, j)$ with the total cofiber of the square

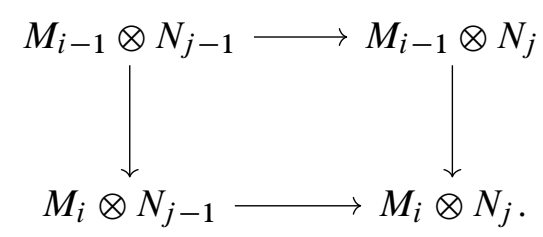

The cofibers of the columns here are $E_{i}^{0} M \otimes N_{j-1}$ and $E_{i}^{0} M \otimes N_{j}$, since the tensor product preserves colimits in each variable, and so the total cofiber $\beta(i, j)$ is isomorphic to the cofiber of the map $E_{i}^{0} M \otimes N_{j-1} \rightarrow E_{i}^{0} M \otimes N_{j}$, which is $E_{i}^{0} M \otimes E_{j}^{0} N$, as required. 
Corollary 3.27 Suppose $A$ is a commutative algebra object of $\operatorname{Seq}(\mathbf{C})$, where $\mathbf{C}$ is as above. Then the adjunction $E^{0} \dashv$ Triv induces an adjunction

$$
E^{0}: \operatorname{Mod}_{A}(\operatorname{Seq}(\mathbf{C})) \rightleftarrows \operatorname{Mod}_{E^{0} A}(\operatorname{Gr}(\mathbf{C})): \text { Triv }
$$

such that $E^{0}$ is symmetric monoidal.

This is immediate from Proposition 3.26 and the following easy formal observation:

Lemma 3.28 Let $\mathbf{C}$ and $\mathbf{D}$ be symmetric monoidal categories, and suppose

$$
F: \mathbf{C} \rightleftarrows \mathbf{D}: G
$$

is an adjunction such that $F$ is symmetric monoidal. If $A$ is a commutative algebra object of $\mathbf{C}$, this induces an adjunction

$$
F_{A}: \operatorname{Mod}_{A}(\mathbf{C}) \rightleftarrows \operatorname{Mod}_{F A}(\mathbf{D}): G_{A}
$$

such that $F_{A}$ is symmetric monoidal.

This allows us to identify the associated graded of a relative tensor product:

Corollary 3.29 Let $\mathbf{C}$ be a symmetric monoidal category compatible with finite colimits that has a zero object. Suppose $A$ is a commutative algebra object in $\mathrm{Seq}(\mathbf{C})$ and that $M$ and $N$ are $A$-modules. Then there is a natural isomorphism

$$
E^{0}\left(M \otimes_{A} N\right) \cong E^{0} M \otimes_{E^{0} A} E^{0} N .
$$

Finally, we check that the colimit of a relative tensor product is the expected one:

Proposition 3.30 Suppose $\mathbf{C}$ is a symmetric monoidal category compatible with small colimits. Then the colimit functor $\operatorname{Seq}(\mathbf{C}) \rightarrow \mathbf{C}$ is symmetric monoidal.

Proof The unit for the tensor product on $\operatorname{Seq}(C)$ is the constant sequence with value $I$, the unit for the tensor product on $\mathbf{C}$. Thus colim preserves the unit. It remains to show that the natural map $\operatorname{colim}_{n}(A \otimes B)_{n} \rightarrow \operatorname{colim}_{n} A_{n} \otimes \operatorname{colim}_{n} B_{n}$ is an isomorphism. But the object $\operatorname{colim}_{n}(A \otimes B)_{n}$ is clearly the colimit over $(i, j) \in \mathbf{N} \times \mathbf{N}$ of $A_{i} \otimes B_{j}$. Since the tensor product on $\mathbf{C}$ preserves colimits in each variable, this colimit is indeed equivalent to $\left(\operatorname{colim}_{i \in \mathbf{N}} A_{i}\right) \otimes\left(\operatorname{colim}_{i \in \mathbf{N}} B_{i}\right)$.

Applying Lemma 3.28, we get: 
Corollary 3.31 Let $\mathbf{C}$ be as above. Suppose $A$ is a commutative algebra object in Seq $(\mathbf{C})$ with colimit $\bar{A}$. Then the colimit-constant adjunction induces an adjunction

$$
\text { colim: } \operatorname{Mod}_{A}(\operatorname{Seq}(\mathbf{C})) \rightleftarrows \operatorname{Mod}_{\bar{A}}(\mathbf{C}) \text { :const }
$$

where the left adjoint is symmetric monoidal.

Corollary 3.32 Suppose $A$ is a commutative algebra object in Seq(C) with colimit $\bar{A}$, and $M$ and $N$ are $A$-modules with colimits $\bar{M}$ and $\bar{N}$. Then colim $M \otimes_{A} N$ is naturally isomorphic to $\bar{M} \otimes_{\bar{A}} \bar{N}$.

\subsection{A Serre spectral sequence for simplicial commutative algebras}

In this subsection we construct a multiplicative "Serre spectral sequence" for the homotopy groups of the cofiber of a cofibration of simplicial commutative algebras. We derive this by studying a spectral sequence for filtered modules over a filtered differential graded algebra. Our spectral sequence has the same form as one constructed by Quillen [25], but his construction does not give the multiplicative structure.

Remark 3.33 We will implicitly assume that all filtrations we consider are nonnegatively graded and exhaustive, in the sense that if $F_{0} A \subseteq F_{1} A \subseteq \cdots$ is a filtration of $A$, then $A$ is the union of the subobjects $F_{i} A$.

Proposition 3.34 Suppose $A$ is a filtered commutative differential graded $k$-algebra, nonnegatively graded, where $k$ is a field, and $B$ and $C$ are filtered $A$-modules, also nonnegatively graded.

(i) If $B$ and $C$ are cofibrant in the model structure on $A$-modules in sequences of maps of chain complexes of Corollary 3.23, then the tensor product $B \otimes_{A} C$ has a canonical filtration with associated graded

$$
E_{*}^{0}\left(B \otimes_{A} C\right) \cong E_{*}^{0} B \otimes_{E_{*}^{0} A} E_{*}^{0} C .
$$

(ii) Suppose $B$ and $C$ are in addition filtered $A$-algebras. Then the filtration of (i) makes $B \otimes_{A} C$ a filtered algebra, so the associated spectral sequence is multiplicative.

Proof Since $k$ is a field, every $k$-module is projective, hence in the projective model structure on the category $\mathrm{Ch}_{k}^{\geq 0}$ of nonnegatively graded chain complexes of $k$-modules every object is cofibrant. Thus in the projective model structure on $\operatorname{Seq}\left(\mathrm{Ch}_{k}^{\geq 0}\right)$ the cofibrant objects are precisely those that are sequences of monomorphisms, ie those that 
correspond to filtered chain complexes. Part (i) then follows from Corollaries 3.24, 3.29 and 3.32 applied to the projective model structure on chain complexes of $k$-modules.

If $B$ and $C$ are filtered $A$-algebras, then we may regard them as associative algebra objects in the category $\operatorname{Mod}_{A}\left(\operatorname{Seq}\left(\mathrm{Ch}_{k}^{\geq 0}\right)\right)$. Their relative tensor product is then also an associative algebra object in this category, and by (i) its underlying object in $\operatorname{Seq}\left(\mathrm{Ch}_{k}^{\geq 0}\right)$ corresponds to a filtered chain complex. Thus $B \otimes_{A} C$ is a filtered algebra, and so yields a multiplicative spectral sequence.

Proposition 3.35 Suppose $A$ is a commutative differential graded $k$-algebra and $B$ and $C$ are $A$-modules, all nonnegatively graded. Filter $A$ and $B$ by degree, and give $C$ the trivial filtration with $F_{p} C=C$ for all $p \geq 0$. Let $B^{\prime}$ and $C^{\prime}$ be cofibrant replacements of $B$ and $C$ as $A$-modules in $\operatorname{Seq}\left(\mathrm{Ch}_{k}^{\geq 0}\right)$. Then in the spectral sequence associated to the induced filtration on $B^{\prime} \otimes_{A} C^{\prime}$ we have:

(i) $E_{s, t}^{1}=\left(B^{\prime} \otimes_{A} \pi_{t-s} C\right)_{s}$, where $A$ acts on $\pi_{*} C$ via the map $A \rightarrow \pi_{0} A$.

(ii) $E_{s, t}^{2}=\pi_{s}\left(B^{\prime} \otimes_{A} \pi_{t-s} C\right)$.

Proof The graded tensor product $E_{*}^{0} B^{\prime} \otimes_{E_{*}^{0} A} E_{*}^{0} C^{\prime}$ has in degree $(s, t)$ the coequalizer of

$$
\bigoplus_{\substack{i j+k=s \\ \rho+\sigma+\tau=t}} E_{i}^{0} B_{\rho}^{\prime} \otimes E_{j}^{0} A_{\sigma} \otimes E_{k}^{0} C_{\tau}^{\prime} \rightrightarrows \bigoplus_{\substack{m+n=s \\ \alpha+\beta=t}} E_{m}^{0} B_{\alpha}^{\prime} \otimes E_{n}^{0} C_{\beta}^{\prime} .
$$

In our case $E_{l}^{0} B_{\gamma}^{\prime}$ and $E_{l}^{0} A_{\gamma}$ are zero unless $l=\gamma$, and $E_{l}^{0} C_{\gamma}^{\prime}$ is zero unless $l=0$, so this is the coequalizer of

$$
\bigoplus_{i+j=s} B_{i}^{\prime} \otimes A_{j} \otimes C_{t-s}^{\prime} \rightrightarrows B_{s}^{\prime} \otimes C_{t-s}^{\prime}
$$

Now observe that the map $A_{j} \otimes C_{t-s}^{\prime} \rightarrow C_{t-s}^{\prime}$ is zero unless $j=0$, since $A_{j}$ is in filtration $j$ and so the product must lie in $\left(E_{j}^{0} C^{\prime}\right)_{t-s}=0$. Thus we can describe this coequalizer as killing all elements of the form $a \cdot b$ with $a \in A$ and $b \in B^{\prime}$, giving $\left(B^{\prime} \otimes_{A} \pi_{0} A\right)_{s} \otimes_{\pi_{0} A} C_{t-s}^{\prime} \cong\left(B^{\prime} \otimes_{\pi_{0} A} C_{t-s}^{\prime}\right)_{s}$.

The differential in $B^{\prime} \otimes_{A} C^{\prime}$ satisfies the Leibniz rule, so if $b \otimes c$ is in filtration $s$ then $d(b \otimes c)=d b \otimes c+b \otimes d c$. Here $d b$ is in lower filtration than $b$, since it is in lower degree, hence $d_{0}$ comes from the differential in $C^{\prime}$. Thus

$$
E_{s, t}^{1} \cong\left(B^{\prime} \otimes_{\pi_{0} A} \pi_{t-s} C\right)_{s} .
$$

Similarly, the next differential $d_{1}$ comes from the differential in $B$, giving

$$
E_{s, t}^{2} \cong \pi_{s}\left(B^{\prime} \otimes_{\pi_{0} A} \pi_{t-s} C\right) .
$$


The Dold-Kan correspondence extends to a Quillen equivalence of model categories (see [28, Section 4]) between simplicial modules and chain complexes, where the weak equivalences in the two categories are the $\pi_{*}$-isomorphisms and the quasiisomorphisms, respectively. Using these model structures we can define derived tensor products as follows:

Definition 3.36 If $A$ is a simplicial graded $\mathbb{F}_{2}$-algebra, $M$ is a right $A$-module, and $N$ is a left $A$-module, then the derived tensor product $M \otimes_{A}^{\mathbb{L}} N$ is the homotopy colimit of the simplicial diagram given by the bar construction, $M \otimes A^{\otimes \bullet} \otimes N$. Similarly, if $A$ is an algebra in chain complexes of graded $\mathbb{F}_{2}$-vector spaces, $M$ is a right $A$-module, and $N$ is a left $A$-module, we define a derived tensor product $M \otimes_{A}^{\mathbb{L}} N$ as the analogous homotopy colimit.

Remark 3.37 In the simplicial case, the homotopy colimit is given by the diagonal of the bar construction.

Remark 3.38 If $M$ is a cofibrant $A$-module, then for any $N$ the derived tensor product $M \otimes_{A}^{\mathbb{L}} N$ is equivalent to the ordinary tensor product $M \otimes_{A} N$.

Lemma 3.39 Let $A$ be a simplicial graded algebra, $X$ a right $A$-module, and $Y$ a left $A$-module. There is a natural quasi-isomorphism

$$
N(X) \otimes_{N(A)}^{\mathbb{L}} N(Y) \rightarrow N\left(X \otimes_{A}^{\mathbb{L}} Y\right) .
$$

Proof There is a natural quasi-isomorphism $N(U) \otimes N(V) \rightarrow N(U \otimes V)$ for all simplicial abelian groups $U$ and $V$. Thus there is a natural transformation of simplicial diagrams $N\left(X \otimes A^{\otimes \bullet} \otimes Y\right) \rightarrow N X \otimes(N A)^{\otimes \bullet} \otimes N Y$ that is a quasi-isomorphism levelwise. This implies that the induced map on homotopy colimits is also a quasiisomorphism.

Corollary 3.40 Suppose given simplicial augmented graded $\mathbb{F}_{2}$-algebras $A, B$ and $C$, and maps $A \rightarrow B$ and $A \rightarrow C$. Then there is a multiplicative spectral sequence

$$
E_{s, t}^{2}=\pi_{s}\left(B \otimes_{A}^{\mathbb{L}} \pi_{t-s}(C)\right) \Rightarrow \pi_{t}\left(B \otimes_{A}^{\mathbb{L}} C\right) .
$$

Proof Let $P$ be a cofibrant replacement for $N B$ as an $N A$-module in $\operatorname{Seq}\left(\mathrm{Ch}_{k}^{\geq 0}\right)$. Then by Proposition 3.35 we have a spectral sequence

$$
E_{s, t}^{2}=\pi_{s}\left(P \otimes_{N A} \pi_{t-s} N C\right) \Rightarrow \pi_{t-s}\left(P \otimes_{N A} N C\right),
$$


which is multiplicative by Proposition 3.34. Since taking the colimit of a sequence is a left Quillen functor, $P$ is also a cofibrant replacement for $N B$ as an $N A$-module, so we can write this as

$$
E_{s, t}^{2}=\pi_{s}\left(N B \otimes_{N A}^{\mathbb{L}} \pi_{t-s} N C\right) \Rightarrow \pi_{t-s}\left(N B \otimes_{N A}^{\mathbb{L}} N C\right) .
$$

By Lemma 3.39 we have a natural quasi-isomorphism $N B \otimes_{N A}^{\mathbb{L}} N C \rightarrow N\left(B \otimes_{A}^{\mathbb{L}} C\right)$ and, since $\pi_{t-s} N C \cong \pi_{t-s} C$ is concentrated in a single degree, a natural quasi-isomorphism $N B \otimes_{N A}^{\mathbb{L}} \pi_{t-s} N C \rightarrow N\left(B \otimes_{A}^{\mathbb{L}} \pi_{t-s} C\right)$. Thus we have a natural isomorphism

$$
E_{s, t}^{2} \cong \pi_{s}\left(B \otimes_{A}^{\mathbb{L}} \pi_{t-s} C\right) \Rightarrow \pi_{t-s}\left(B \otimes_{A}^{\mathbb{L}} C\right) .
$$

As observed by Turner [32, Proof of Lemma 3.1], this spectral sequence can be used to get a "Serre spectral sequence" for cofibration sequences of simplicial commutative algebras:

Corollary 3.41 ("Serre spectral sequence") Suppose $f: A \rightarrow B$ is a cofibration of simplicial augmented graded commutative $\mathbb{F}_{2}$-algebras with cofiber $C$ and $\pi_{0} A=\mathbb{F}_{2}$. Then there is a multiplicative spectral sequence

$$
\pi_{s}(C) \otimes_{\mathbb{F}_{2}} \pi_{t-s}(A) \Rightarrow \pi_{t}(B) .
$$

Proof By Corollary 3.40 there is a multiplicative spectral sequence

$$
E_{s, t}^{2}=\pi_{s}\left(B \otimes_{A}^{\mathbb{L}} \pi_{t-s} A\right) \Rightarrow \pi_{t}(B) .
$$

By definition $C \cong B \otimes_{A} \mathbb{F}_{2}$, and so

$$
C \otimes_{\mathbb{F}_{2}} \pi_{t} A \cong\left(B \otimes_{A} \mathbb{F}_{2}\right) \otimes_{\mathbb{F}_{2}} \pi_{t} A \cong B \otimes_{A} \pi_{t} A,
$$

which is isomorphic to $B \otimes \mathbb{L}_{A} \pi_{t} A$ since $A \rightarrow B$ is a cofibration and the model structure on simplicial commutative algebras is left proper by Theorem 3.4. Since $\mathbb{F}_{2}$ is a field we have

$$
\pi_{s}\left(C \otimes_{\mathbb{F}_{2}} \pi_{t-s} A\right) \cong \pi_{s} C \otimes_{\mathbb{F}_{2}} \pi_{t-s} A,
$$

and so we can rewrite the $E^{2}$-term of the spectral sequence as

$$
E_{s, t}^{2} \cong \pi_{s}(C) \otimes_{\mathbb{F}_{2}} \pi_{t-s}(A)
$$




\section{Derived functors of $U$}

Our goal in this section is to compute $\pi_{*} U(M)$ where $M$ is a simplicial unstable $A-$ module. As a simplicial commutative algebra, $U(M)$ depends only on the top nonzero Steenrod operations in $M$; in Section 4.1 we consider graded vector spaces equipped with only these operations, which we call restricted vector spaces, and observe that a simplicial restricted vector space decomposes up to weak equivalence as a coproduct of simple pieces. In Section 4.2 we compute the derived functors of $U$ for these simpler objects, which gives a description of $\pi_{*} U(M)$ as a graded commutative algebra with higher divided square operations. Using this we then give a more functorial description of $\pi_{*} U(M)$ in Section 4.3, which in particular lets us identify the action of the Steenrod operations.

\subsection{Restricted vector spaces}

In this subsection we define restricted vector spaces and make some observations about their structure; in particular, we show that a chain complex of restricted vector spaces always decomposes up to quasi-isomorphism as a direct sum of certain very simple complexes.

Definition 4.1 A restricted vector space (over $\mathbb{F}_{2}$ ) is a nonnegatively graded vector space $V$ equipped with linear maps $\phi_{i}: V^{i} \rightarrow V^{2 i}$ for all $i$, called the restriction maps of $V$, such that $\phi_{0}: V^{0} \rightarrow V^{0}$ is the identity. A homomorphism of restricted vector spaces $f: V \rightarrow W$ is a homomorphism of graded vector spaces such that $\phi_{i} f^{i}=f^{2 i} \phi_{i}$ for all $i$. We write Restr for the category of restricted vector spaces and restricted vector space homomorphisms. This is an abelian category.

Definition 4.2 For $n \leq 0$, let $F(n)$ be the free restricted vector space with one generator $\iota_{n}$ in degree $n$. Thus $F(n)^{2^{r} n}=\mathbb{F}_{2}$ with $\phi_{2} r_{n}=$ id and $F(n)^{i}=0$ otherwise; in particular $F(0)$ is just $\mathbb{F}_{2}$ in degree 0 .

For $k, n>0$, let $T(n, k)$ be the nilpotent restricted vector space with one generator $\iota_{n, k}$ in degree $n$ subject to $\phi^{k} \iota_{n, k}=0$; that is, $T(n, k)=F(n) / \phi^{k}$. Thus $T(n, k)^{2^{r} n}=\mathbb{F}_{2}$ for $r=0, \ldots, k$ with $\phi_{2^{r} n}=\mathrm{id}$ for $r=0, \ldots, k-1$, and $T(n, k)^{i}$ is 0 otherwise.

Definition 4.3 Let $V$ be a restricted vector space. A basis $S$ of $V$ consists of sets $S^{i}$ of elements of $V^{i}$ such that $S^{0}$ is a basis for $V^{0}$ and if $i=2^{r} p$ with $p$ odd, then the set $\left(S^{2^{r} p} \cup \phi\left(S^{2^{r-1} p}\right) \cup \cdots \cup \phi^{r}\left(S^{p}\right)\right) \backslash\{0\}$ is a basis for $V^{i}$.

Remark 4.4 It is clear that any restricted vector space has a basis, since we can inductively choose complements of $\phi\left(V_{i}\right)$ in $V_{2 i}$. Equivalently, any restricted vector space decomposes as a direct sum of copies of $F(n)$ and $T(n, k)$. 
Definition 4.5 Let $C(q)$ be the nonnegatively graded chain complex of restricted vector spaces

$$
\cdots \rightarrow 0 \rightarrow 0 \rightarrow F(q)
$$

with $F(q)$ in degree 0 , and let $C(q, k)$ be the chain complex

$$
\cdots \rightarrow 0 \rightarrow F\left(2^{k} q\right) \hookrightarrow F(q),
$$

with $F(q)$ in degree 0 and $F\left(2^{k} q\right)$ in degree 1 .

Given a chain complex $C$, we denote by $C[n]$ the suspended chain complex with $C[n]_{i}=C_{i-n}$. Then clearly

$$
\begin{gathered}
\mathrm{H}_{*}(C(q)[n]) \cong\left\{\begin{array}{cl}
F(q) & \text { if } *=n, \\
0 & \text { if } * \neq n,
\end{array}\right. \\
\mathrm{H}_{*}(C(q, k)[n]) \cong\left\{\begin{array}{cl}
T(q, k) & \text { if } *=n, \\
0 & \text { if } * \neq n .
\end{array}\right.
\end{gathered}
$$

Proposition 4.6 Any chain complex of restricted vector spaces is quasi-isomorphic to a direct sum of copies of $C(q)[n]$ and $C(q, k)[n]$.

Proof Let $\left(V_{*}, d\right)$ be a chain complex of restricted vector spaces. Pick a basis $S_{i}$ of $\mathrm{H}_{i}\left(V_{*}\right)$. For $v \in S_{i}^{q}$ define $W_{v}$ to be $F(q)[i]$ if $\phi^{r} v$ is never zero, and $C(q, k)[i]$ if $\phi^{k} v=0$ but $\phi^{r} v \neq 0$ for $r<k$. Let $\hat{v}$ be a lift of $v$ to $V_{i}$; in the first case $\hat{v}$ defines a map $\psi_{v}: W_{v} \rightarrow V_{\bullet}$. In the second case, since $\phi^{k}(v)=0$ we can pick $\hat{w} \in V_{i+1}$ such that $d(\widehat{w})=\phi^{k}(\widehat{v})$; then $\hat{v}$ and $\widehat{w}$ define a map $\psi_{v}$ from $W_{v}$ to $V_{\bullet}$. Let $W:=\bigoplus_{v \in S} W_{v}$ and let $\psi: W \rightarrow V$ be $\bigoplus_{v \in S} \psi_{v}$. Then $\psi$ is a quasi-isomorphism, since it is clear that on homology $\psi_{v}$ induces the inclusion in $\mathrm{H}_{i}\left(V_{\bullet}, d\right)$ of the subspace generated by $v$.

By the Dold-Kan correspondence the category $\mathrm{Ch}(\mathrm{Restr})_{\geq 0}$ of nonnegatively graded chain complexes of restricted vector spaces is equivalent to the category of simplicial restricted vector spaces. Let's write $K[n, q]$ and $K[n, q, k]$ for the simplicial objects corresponding to $F(q)[n]$ and $C(q, k)[n]$, respectively, under this equivalence; then Proposition 4.6 corresponds to:

Corollary 4.7 Any simplicial restricted vector space is weakly homotopy equivalent to a coproduct of copies of $K[n, q]$ and $K[n, q, k]$. 


\subsection{Computation of the derived functors of $U$}

In this subsection we will prove the main technical result of this paper: we compute the homotopy groups of the free unstable $A$-algebra on a simplicial unstable $A$-module. As an algebra, $U(V)$ depends only on $V$ as a restricted vector space: it is the "enveloping algebra" of $V$, given by the free graded commutative algebra on $V$ subject to the relation $x^{2}=\phi(x)$, ie $S(V) /\left(x^{2}=\phi(x)\right)$, where $S(V)$ is the graded symmetric algebra on $V$. If $V$ is a simplicial restricted vector space we may ask about $\pi_{*}(U V)$. It does not depend functorially on $\pi_{*}(V)$; we do not have Dold's Theorem 3.15 working for us. We will describe $\pi_{*}(U V)$ in terms of $\pi_{*}(V)$, but not functorially. Our description will use the functor $\mathfrak{S}$, given to us by Dold's theorem, such that $\pi_{*}(S V)=\mathfrak{S}\left(\pi_{*}(V)\right)$. It is described in detail above, in Theorem 3.17. We will also use the "loops" functor $\Omega$ and its first derived functor $\Omega_{1}$, defined by the exact sequence

$$
0 \rightarrow \Sigma \Omega_{1} V \rightarrow \Phi V \stackrel{\phi}{\rightarrow} V \rightarrow \Sigma \Omega V \rightarrow 0 .
$$

where $\Phi$ denotes the "doubling" functor, $(\Phi V)_{n}^{2 q}=V_{n}^{q}$ and $(\Phi V)_{n}^{2 q+1}=0$.

Here is the result:

Theorem 4.8 If $V$ is a simplicial restricted vector space, then there is a (noncanonical) isomorphism

$$
\pi_{*} U V \cong U\left(\pi_{0} V\right)[0] \otimes \mathfrak{S}\left(\Sigma \Omega \pi_{*>0} V\right) \otimes \mathfrak{S}\left(\left(\Sigma \Omega_{1} \pi_{*>0} V\right)[1]\right),
$$

where [1] denotes a shift by 1 in the simplicial degree. (By noncanonical we mean that the isomorphism depends on a choice of basis of $\pi_{*} V$.)

By Corollary 4.7 we know that any simplicial restricted vector space is weakly equivalent to a coproduct of copies of $K[n, q]$ and $K[n, q, k]$. To show that this carries over to a decomposition of $U(M)$ up to weak equivalence, we observe $U$ preserves weak equivalences and colimits:

Proposition 4.9 $U$, considered as a functor from restricted vector spaces to graded commutative $\mathbb{F}_{2}$-algebras, preserves colimits and weak equivalences.

Proof We first show that $U$ preserves colimits. Let $U^{\prime}$ denote $U$, regarded as a functor from restricted vector spaces to augmented graded commutative $\mathbb{F}_{2}$-algebras. The forgetful functor from augmented algebras to algebras preserves colimits, so it suffices to show that $U^{\prime}$ preserves colimits. But this is clear since $U^{\prime}$ has a right adjoint, namely the augmentation ideal functor for augmented graded commutative 
$\mathbb{F}_{2}$-algebras, regarded as a functor to restricted vector spaces with restriction maps given by squaring.

To see that $U$ preserves weak equivalences, consider the word-length filtration on $U(V)$ for $V$ a simplicial restricted vector space. This gives rise to a spectral sequence of the form

$$
\pi_{t} E_{S}(V)=\mathfrak{E}_{S}\left(\pi_{*} V\right)_{t} \Rightarrow \pi_{t} U(V),
$$

where $\mathfrak{E}$ is as in Section 3.2 Moreover, since the filtration is natural in $V$, so is the spectral sequence. Thus a weak equivalence $f: V \rightarrow W$ of simplicial restricted vector spaces induces a morphism of spectral sequences that gives an isomorphism on the $E^{1}$-page, since this only depends on the homotopy of the simplicial restricted vector space. This implies that the map is an isomorphism of spectral sequences and hence, as these spectral sequences converge, it follows that $U(f)$ is a weak equivalence of simplicial graded commutative algebras.

Combining this with Corollary 4.7 we see that $U(M)$, for any simplicial restricted vector space $M$, is weakly equivalent to a tensor product of copies of $U(K[n, q])$ and $U(K[n, q, k])$. It thus suffices to prove Theorem 4.8 in these two cases. We begin with the easiest case, namely $\pi_{*} U(K[n, q])$ for $q>0$. For this we need to recall the explicit form of the Dold-Kan construction:

Definition 4.10 Let $\mathbf{C}$ be an abelian category. The Dold-Kan construction

$$
K: \operatorname{Ch}(\mathbf{C})_{\geq 0} \rightarrow \operatorname{Fun}\left(\Delta^{\mathrm{op}}, \mathbf{C}\right)
$$

sends a nonnegatively graded chain complex $A$ to the simplicial object $K(A)$ defined as follows: We set

$$
K(A)_{n}=\bigoplus_{\alpha:[n] \rightarrow[k]} A_{k},
$$

where the coproduct is over surjective maps out of $[n]$. Then a map $K(A)_{n} \rightarrow K(A)_{m}$ is described by a "matrix" of maps $f_{\alpha, \beta}: A_{k} \rightarrow A_{l}$ from the component corresponding to $\alpha:[n] \rightarrow[k]$ to the component corresponding to $\beta:[m] \rightarrow[l]$. To define the map $\phi^{*}: K(A)_{n} \rightarrow K(A)_{m}$ corresponding to $\phi:[m] \rightarrow[n]$ in $\boldsymbol{\Delta}$ we take this to be given by

$$
f_{\alpha, \beta}:= \begin{cases}\text { id } & \text { if } l=k \text { and } \beta=\alpha \phi, \\ d & \text { if } l=k-1 \text { and } d^{0} \beta=\alpha \phi, \\ 0 & \text { otherwise }\end{cases}
$$

The Dold-Kan correspondence (see Dold [7], Dold and Puppe [8], Kan [17]) is then that the functor $K$ is an equivalence of categories, with inverse the normalized chain complex functor. 
Lemma 4.11 Let $\mathbb{F}_{2}[n, q]$ denote the chain complex of graded vector spaces that is 0 except in the degree $n$, where it is $\mathbb{F}_{2}[q]$ (the graded vector space with $\mathbb{F}_{2}$ in degree $q$ and 0 elsewhere). Then for $q>0$ we have $U K[n, q] \cong S\left(K \mathbb{F}_{2}[n, q]\right)$, where $K \mathbb{F}_{2}[n, q]$ is the Dold-Kan construction for graded vector spaces applied to $\mathbb{F}_{2}[n, q]$. In particular, we have an isomorphism

$$
\pi_{*} U K[n, q] \cong \mathfrak{S F}_{2}[n, q]
$$

for all $n$ and $q>0$.

Proof From the definition of the Dold-Kan functor $K$ we have

$$
K[n, q]_{i}=\bigoplus_{[i] \rightarrow[n]} F(q)
$$

and so for $q>0$ we have

$$
U K[n, q]_{i} \cong \bigotimes_{[i] \rightarrow[n]} U F(q) \cong \bigotimes_{[i] \rightarrow[n]} S \mathbb{F}_{2}[q] \cong S\left(\bigoplus_{[i] \rightarrow[n]} \mathbb{F}_{2}[q]\right) \cong S\left(K \mathbb{F}_{2}[n, q]\right)_{i}
$$

Moreover, the simplicial structure maps in $U K[n, q]$ and $S K \mathbb{F}_{2}[n, q]$ are also clearly the same (on "components" they are either the identity or zero), so these simplicial graded vector spaces are isomorphic.

For the case $q=0$ the algebra $U F(0)$ is not a symmetric algebra, since we impose the relation $x^{2}=x$ on the generator $x$ : it is a Boolean algebra. If $V$ is a vector space, we write $b(V)$ for the free Boolean algebra $s(V) /\left(x^{2}=x\right)$ on $V$ (where $s(V)$ is the ungraded symmetric algebra on $V$ ). By Theorem 3.15, there is a functor $\mathfrak{b}$ : grVect $\rightarrow$ grVect such that $\pi_{*} b(V)=\mathfrak{b}\left(\pi_{*} V\right)$ for $V$ a simplicial vector space.

Lemma 4.12 Suppose $V$ is a graded $\mathbb{F}_{2}$-vector space. Then

$$
\mathfrak{b}(V)_{*}=\left\{\begin{array}{cl}
b\left(V_{0}\right) & \text { if } *=0, \\
0 & \text { otherwise. }
\end{array}\right.
$$

Proof Suppose $V$ is a simplicial vector space. Then Theorem 3.9 implies that for any element $a$ in degree $n>0$ in the chain complex associated to $b(V)$ such that $d a=0$, there exists an element $\delta_{1} a$ in degree $n+1$ such that $d \delta_{1} a=\phi(a)=a$. Thus $\pi_{*} b(V)=0$ for $*>0$.

Lemma 4.13 For any $n$ we have an isomorphism $U K[n, 0] \cong b\left(K \mathbb{F}_{2}[n]\right)[0]$, the simplicial graded vector space with $b\left(K \mathbb{F}_{2}[n]\right)$ in degree 0 , and so

$$
\pi_{*} U K[n, 0]= \begin{cases}\left(b\left(\mathbb{F}_{2}\right)\right)[0] & \text { if } n=0 \\ b(0)[0] \cong \mathbb{F}_{2}[0] & \text { if } n \neq 0 .\end{cases}
$$


Proof As in the proof of Lemma 4.11 we have

$$
U K[n, 0]_{i} \cong \bigotimes_{[i] \rightarrow[n]} U F(0) \cong \bigotimes_{[i] \rightarrow[n]} b\left(\mathbb{F}_{2}\right)[0] \cong b\left(\bigoplus_{[i] \rightarrow[n]} \mathbb{F}_{2}\right)[0] \cong b\left(K \mathbb{F}_{2}[n]\right)[0]_{i},
$$

and the simplicial structure maps are again the same.

Now we consider the consider the more complicated case, namely $U K[n, q, k]$. There is a cofibration sequence

$$
F(q)[n] \rightarrow C(q, k)[n] \rightarrow F\left(2^{k} q\right)[n+1]
$$

of chain complexes of restricted vector spaces. By the Dold-Kan correspondence this gives a cofibration sequence

$$
K[n, q] \rightarrow K[n, q, k] \rightarrow K\left[n+1,2^{k} q\right]
$$

of simplicial restricted vector spaces, and so a cofibration sequence

$$
U(K[n, q]) \rightarrow U(K[n, q, k]) \rightarrow U\left(K\left[n+1,2^{k} q\right]\right)
$$

of simplicial commutative $\mathbb{F}_{2}$-algebras by Proposition 4.9. We want to apply the Serre spectral sequence of Corollary 3.41 to this cofibration sequence to compute $\pi_{*} U(K[n, q, k])$; to do this we first observe that the map $U(K[n, q]) \rightarrow U(K[n, q, k])$ is a cofibration of simplicial graded commutative algebras:

Lemma 4.14 The map $U(K[n, q]) \rightarrow U(K[n, q, k])$ is almost-free in the sense of Definition 3.5, and so is a cofibration of simplicial graded commutative algebras.

Proof We have

$$
K[n, q, k]_{i}=\bigoplus_{[i] \rightarrow[n]} F(q) \oplus \bigoplus_{[i] \rightarrow[n+1]} F\left(2^{k} q\right)
$$

and $U K[n, q, k]_{i} \cong U K[n, q]_{i} \otimes S\left(V_{i}\right)$ where $V_{i}=\bigoplus_{[i] \rightarrow[n+1]} \mathbb{F}_{2}\left[2^{k} q\right]$. It is clear that the simplicial structure maps $\psi^{*}$ take $U K[q, n]_{*}$ to itself, and are induced by maps between the $V_{n}$ except when $\psi$ is such that for some $\beta$ and $\gamma$ we have $\beta \psi=d^{0} \gamma$, since this is the case when the differential in the chain complex occurs in the definition of $\psi^{*}$ for $K[n, q, k]$.

But the map $\beta:[i] \rightarrow[n+1]$ is surjective and order-preserving, so it must send 0 to 0 . Thus $\beta \psi$ will hit 0 in $[n+1]$ for all $\beta$ if $\psi$ hits 0 in $[i]$, in which case $\beta \psi$ cannot be of the form $d^{0} \gamma$. This is clearly the case for the degeneracies $s^{j}:[i+1] \rightarrow[i]$ for all $j$ (as they are surjective) and the face maps $d^{j}:[i-1] \rightarrow[i]$ for $j \neq 0$. Thus the 
structure maps of $U(K[n, q, k])$ corresponding to all degeneracies and all face maps other than $d_{0}$ are induced from maps between the $V_{i}$.

Proposition 4.15 The Serre spectral sequence for the cofibration sequence

$$
U(K[n, q]) \stackrel{\alpha}{\rightarrow} U(K[n, q, k]) \stackrel{\beta}{\rightarrow} U\left(K\left[n+1,2^{k} q\right]\right)
$$

collapses at the $E^{2}$-page.

Proof By Corollary 3.41 the spectral sequence in question is a multiplicative spectral sequence of the form

$$
E_{s, t}^{2}=\pi_{S}\left(U K\left[n+1,2^{k} q\right]\right) \otimes \pi_{t-s}(U K[n, q]) \Rightarrow \pi_{t}(U K[n, q, k]),
$$

with differentials $d^{r}: E_{s, t}^{r} \rightarrow E_{s-r, t-1}^{r}$. Write $\iota$ to denote the fundamental class in $\pi_{n} K[n, q]=E_{0, n}^{2}$ and $\kappa$ for the fundamental class in $\pi_{n+1} K\left[n+1,2^{k} q\right]=E_{n+1, n+1}^{2}$. Since the spectral sequence is multiplicative, it suffices to show that there are no nonzero differentials on the classes $\delta_{I} \iota$ and $\delta_{I} \kappa$. Clearly there are no possible nonzero differentials on $\delta_{I} \iota$, and the only differential on $\kappa$ that hits a nonzero group is $d^{n+1}$, but $d^{n+1} \kappa$ cannot be $\iota$ since they differ in internal grading. Moreover, the groups that might support differentials hitting $\kappa$ are all zero, so $\kappa$ must survive to $E^{\infty}$.

There is an obvious map of cofibration sequences from

$$
U(K[n, q]) \rightarrow U(K[n, q, k]) \rightarrow U\left(K\left[n+1,2^{k} q\right]\right)
$$

to

$$
\mathbb{F}_{2}[0] \rightarrow U\left(K\left[n+1,2^{k} q\right]\right) \stackrel{\mathrm{id}}{\longrightarrow} U\left(K\left[n+1,2^{k} q\right]\right),
$$

and the map $\mathbb{F}_{2}[0] \rightarrow U\left(K\left[n+1,2^{k} q\right]\right)$ is a cofibration since $U\left(K\left[n+1,2^{k} q\right]\right)$ is free. Thus we get a morphism of spectral sequences, given on the $E^{2}$-page by projection to $\pi_{*} U\left(K\left[n+1,2^{k} q\right]\right)$ and on the $E^{\infty}$-page by $\pi_{*} \beta$. This means that $\pi_{*} \beta$ must send $\kappa$ to the fundamental class $\kappa^{\prime}$ in $\pi_{*} U\left(K\left[n+1,2^{k} q\right]\right)$, and so for any admissible sequence $I$ the class $\delta_{I} \kappa$ is mapped to $\delta_{I} \kappa^{\prime}$, which is nonzero. This implies that $\delta_{I} \kappa$ must also survive to $E^{\infty}$ for all $I$. By multiplicativity, this means the spectral sequence has no nonzero differentials, ie it collapses on the $E^{2}$-page.

Corollary 4.16 There is an isomorphism

$$
\pi_{*} U(K[n, q, k]) \cong \mathfrak{S F}_{2}[n, q] \otimes \mathfrak{S F}_{2}\left[n+1,2^{k} q\right]
$$

of algebras over the triple $\mathfrak{S}$. 
Proof The map $\pi_{*} \beta: \pi_{*} U K[n, q, k] \rightarrow \pi_{*} U K\left[n+1,2^{k} q\right]$ is surjective, as we saw in the proof of Proposition 4.15. Since $\pi_{*} U K\left[n+1,2^{k} q\right]$ is free, choosing a preimage of the generator gives a map $\pi_{*} U K\left[n+1,2^{k} q\right] \rightarrow \pi_{*} U K[n, q, k]$ of $\mathfrak{S}$-algebras. Since the tensor product is the coproduct, we get a map

$$
\pi_{*} U K[n, q] \otimes \pi_{*} U K\left[n+1,2^{k} q\right] \rightarrow \pi_{*} U K[n, q, k]
$$

of $\mathfrak{S}$-algebras. Filter the left-hand side by degree and the right-hand side by the filtration from the Serre spectral sequence. The collapse of this spectral sequence implies that this gives an isomorphism of the graded objects associated to the filtration, hence this map is an isomorphism of bigraded vector spaces and so also an isomorphism of $\mathfrak{S}$-algebras.

Combining Lemmas 4.11 and 4.13 and Corollary 4.16 now completes the proof of Theorem 4.8. Applying this to the $E_{2}$-term of our spectral sequence, we deduce the following:

\section{Corollary 4.17}

(i) If $E$ is a connected spectrum of finite type, the $E_{2}$-term of the spectral sequence for $\mathrm{H}^{*} \Omega^{\infty} E$ is of the form

$$
E_{2} \cong U D\left(\mathrm{H}^{*} E\right)[0] \otimes \mathfrak{S}\left(\Sigma \Omega \mathbb{L}_{*>0} D\left(\mathrm{H}^{*} E\right)\right) \otimes \mathfrak{S}\left(\Sigma \Omega_{1} \mathbb{L}_{*>0} D\left(\mathrm{H}^{*} E\right)[1]\right) .
$$

(ii) If in addition the top squares in $\mathbb{L}_{*} D\left(\mathrm{H}^{*} E\right)$ are all zero for $*>0$, then the $E_{2}$-term is given by

$$
E_{2} \cong U D\left(\mathrm{H}^{*} E\right)[0] \otimes \mathfrak{E}\left(\mathbb{L}_{*>0} D\left(\mathrm{H}^{*} E\right)\right) .
$$

\subsection{A functorial description of the derived functors}

The description of $\pi_{*} U M$ for a simplicial restricted vector space we obtained above is compatible with the products and $\delta$-operations. However, in the case of interest, $M$ is the underlying simplicial restricted vector space of a simplicial unstable $A$-module; this means that there are also Steenrod operations on $\pi_{*} U M$. We will now give a more functorial description of $\pi_{*} U M$ that is also compatible with these operations.

If $M$ is a simplicial unstable $A$-module, we have a natural transformation $M \rightarrow \Sigma \Omega M$, which induces a map $\pi_{*} U M \rightarrow \pi_{*} U \Sigma \Omega M$. Since the top squares in $\Sigma \Omega M$ are all zero, as a simplicial commutative algebra $U \Sigma \Omega M$ is isomorphic to $E \Sigma \Omega M$, and hence $\pi_{*} U \Sigma \Omega M$ is isomorphic to $\mathfrak{E}\left(\pi_{*} \Sigma \Omega M\right)$. We can also easily describe the action of the Steenrod operations here, using the following observation of Dwyer: 
Proposition 4.18 [9, Proposition 2.7] Let $R$ be a simplicial unstable algebra over the Steenrod algebra; then $\pi_{*} R$ supports both higher divided squares and Steenrod operations. These are related as follows:

$$
\mathrm{Sq}^{k} \delta_{i}= \begin{cases}0 & \text { for } k \text { odd } \\ \delta_{i} \mathrm{Sq}^{k / 2} & \text { for } k \text { even, }\end{cases}
$$

for $i \geq 2$. Moreover, if all squares in $R$ are zero, then the same is true for $i=1$.

Proof Write $\mathrm{Sq}:=\mathrm{Sq}^{0}+\mathrm{Sq}^{1}+\cdots$. By the Cartan formula, Sq: $R \rightarrow R$ is an algebra homomorphism. Since the operation $\delta_{i}$ is natural, this means $\delta_{i} \mathrm{Sq}=\mathrm{Sq} \delta_{i}$ in $\pi_{*} R$. Considering the homogeneous parts in each internal degree on both sides gives the result.

We first consider the case where $M$ is a simplicial unstable $A$-module such that $\pi_{0} M=0$ :

Proposition 4.19 Suppose $M$ is a levelwise projective simplicial unstable $A$-module such that $\pi_{0} M=0$. Then there is a natural isomorphism of commutative bigraded $\mathbb{F}_{2}$-algebras

$$
\pi_{*} U M \rightarrow \mathfrak{S}\left(\pi_{*} \Sigma \Omega M\right),
$$

compatible with Steenrod operations and higher divided squares.

Proof Observe that if $N$ is a simplicial unstable $A$-module such that $\pi_{0} N=0$ and the top squares in $N$ vanish (such as $N=\Sigma \Omega M$ ), then we have an isomorphism $\mathfrak{E}\left(\pi_{*} N\right) \cong \mathfrak{S}\left(\pi_{*} N\right) \otimes \mathfrak{S}\left(\delta_{1} \pi_{*} N\right)$, compatible with the Steenrod operations. Moreover, the inclusion $\mathfrak{S}\left(\pi_{*} N\right) \hookrightarrow \mathfrak{E}\left(\pi_{*} N\right)$ and retraction $\mathfrak{E}\left(\pi_{*} N\right) \rightarrow \mathfrak{S}\left(\pi_{*} N\right)$ are compatible with Steenrod operations. Taking $N=\Sigma \Omega M$ we thus have a natural map of graded algebras

$$
\pi_{*} U M \rightarrow \mathfrak{E}\left(\pi_{*} \Sigma \Omega M\right) \rightarrow \mathfrak{S}\left(\pi_{*} \Sigma \Omega M\right),
$$

compatible with Steenrod squares and $\delta$-operations. We will show that this map is an isomorphism.

$M$ is weakly equivalent to a coproduct of copies of $K[n, q]$ and $K[n, q, k]$, by Corollary 4.7. We know $U$ preserves weak equivalences and colimits by Proposition 4.9, and $\Omega$ preserves coproducts and weak equivalences between levelwise projective objects. Thus it suffices to prove the result when $M$ is $K[n, q]$ or $K[n, q, k]$.

In the first case, $\Sigma \Omega K[n, q]$ is $K\left(\mathbb{F}_{2}[n, q]\right)$, and by Lemma 4.11 we know that $U K[n, q]$ is $S K\left(\mathbb{F}_{2}[n, q]\right)$ for $n>0$, so the map $U K[n, q] \rightarrow U \Sigma \Omega K[n, q]$ is the 
natural map $S K\left(\mathbb{F}_{2}[n, q]\right) \rightarrow E K\left(\mathbb{F}_{2}[n, q]\right)$. On homotopy this is just the inclusion of the factor $\mathfrak{S}\left(\mathbb{F}_{2}[n, q]\right)$.

When $n=0$, we have $\Sigma \Omega K[n, 0] \simeq 0$, so

$$
\pi_{*} U K[n, 0] \rightarrow \pi_{*} U \Sigma \Omega K[n, 0] \rightarrow \mathfrak{S}\left(\pi_{*} \Sigma \Omega K[n, 0]\right)
$$

is the identity map on $\mathbb{F}_{2}[0]$ by Lemma 4.13 .

For $U K[n, q, k]$ we consider the extension sequence

$$
U K[n, q] \rightarrow U K[n, q, k] \rightarrow U K\left[n+1,2^{k} q\right] .
$$

On homotopy, this leads to a commutative diagram

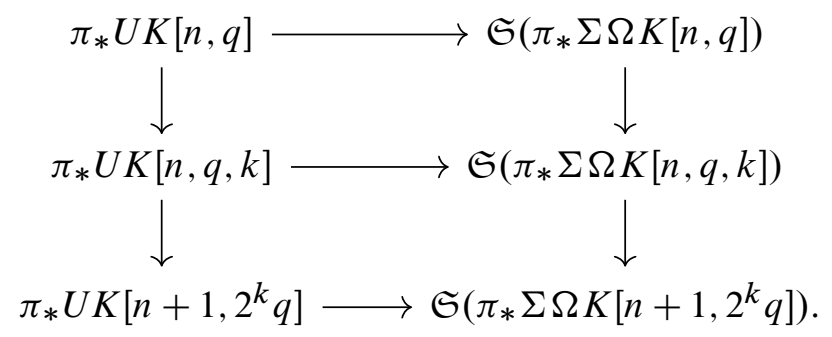

Here we have already shown that the top and bottom horizontal morphism are isomorphisms. But the chain complex $\Sigma \Omega C(q, k)$ is clearly $\mathbb{F}_{2}[q] \oplus \Sigma \mathbb{F}_{2}\left[2^{k} q\right]$, so the right vertical maps are a split extension sequence. Moreover, from the proof of Corollary 4.16 we know that the lower left vertical map is surjective, and that choosing a preimage of the generator gives an isomorphism

$$
\mathfrak{S}\left(\pi_{*} \Sigma \Omega K[n, q]\right) \otimes \mathfrak{S}\left(\pi_{*} \Sigma \Omega K\left[n+1,2^{k} q\right]\right) \stackrel{\sim}{\rightarrow} \pi_{*} U K[n, q, k] .
$$

The composite of this with the map $\pi_{*} U K[n, q, k] \rightarrow \mathfrak{S}\left(\pi_{*} \Sigma \Omega K[n, q, k]\right)$ is also an isomorphism (since it is determined by where it sends the generators). Thus by the 2-out-of-3 property the middle horizontal map here must also be an isomorphism, which completes the proof.

For a general simplicial unstable $A$-module $M$ we have a projection $M \rightarrow \pi_{0} M[0]$. Writing $M_{>0}$ for the fiber of this map, we have a pushout square

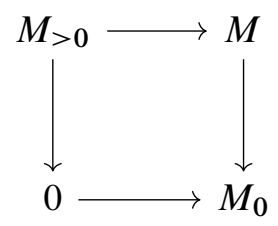

where $M_{0}$ is weakly equivalent to $\pi_{0} M[0]$ (as can be seen from the long exact sequence 
in homotopy groups). Thus we have a pushout diagram

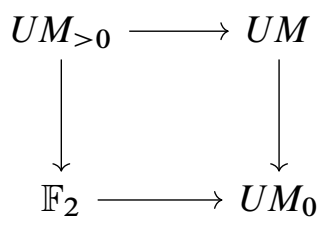

of simplicial unstable $A$-algebras. On homotopy we thus have maps

$$
\pi_{*} U M_{>0} \rightarrow \pi_{*} U M \rightarrow U \pi_{0} M[0],
$$

where the second map is an isomorphism on $\pi_{0}$. We thus have a canonical map $U \pi_{0} M[0] \rightarrow \pi_{*} U M$, and since the tensor product is the coproduct here we get a map

$$
\pi_{*} U M_{>0} \otimes U \pi_{0} M[0] \rightarrow \pi_{*} U M,
$$

compatible with all the operations in play. Moreover, this is an isomorphism; as usual, this follows from considering the case where $M$ is $K[n, q]$ or $K[n, q, k]$.

Theorem 4.20 Suppose $M$ is a simplicial unstable $A$-module, and let $M^{\prime} \rightarrow M$ be a weak equivalence where $M^{\prime}$ is levelwise projective. Then we have a natural isomorphism of commutative bigraded $\mathbb{F}_{2}$-algebras

$$
\mathfrak{S}\left(\pi_{*>0} \Sigma \Omega M^{\prime}\right) \otimes U \pi_{0} M[0] \stackrel{\sim}{\longrightarrow} \pi_{*} U M,
$$

compatible with Steenrod operations and higher divided squares. Moreover, there is a short exact sequence

$$
0 \rightarrow \Sigma \Omega_{1} \pi_{*>0} M[1] \rightarrow \pi_{*>0} \Sigma \Omega M^{\prime} \rightarrow \Sigma \Omega \pi_{*>0} M \rightarrow 0
$$

of graded unstable $A$-modules.

Proof Since $U$ preserves weak equivalences by Proposition 4.9, $\pi_{*} U M^{\prime} \cong \pi_{*} U M$, so the desired isomorphism follows from Proposition 4.19. The short exact sequence is a consequence of the hyperhomology spectral sequence

$$
\Sigma \Omega_{s} \pi_{t} M \Rightarrow \pi_{s+t} \Sigma \Omega M
$$

(see for example [14]), which collapses.

Corollary 4.21 Let $X$ be a connected spectrum of finite type. In the infinite loops spectral sequence for $X$ the $E_{2}$-term is isomorphic to

$$
\mathfrak{S}\left(\mathbb{L}_{*>0}(\Sigma \Omega D)\left(\mathrm{H}^{*} X\right)\right) \otimes U D\left(\mathrm{H}^{*} X\right),
$$

compatibly with products, Steenrod operations, and higher divided squares. Moreover, 
there is a short exact sequence

$$
0 \rightarrow \Sigma \Omega_{1} \mathbb{L}_{*>0} D\left(\mathrm{H}^{*} X\right)[1] \rightarrow \mathbb{L}_{*>0} \Sigma \Omega D\left(\mathrm{H}^{*} X\right) \rightarrow \Sigma \Omega \mathbb{L}_{*>0} D\left(\mathrm{H}^{*} X\right) \rightarrow 0
$$

of graded unstable $A$-modules.

Proof Recall the $E_{2}$-term is obtained by applying $U D$ to a free simplicial resolution of $\mathrm{H}^{*} X$ as an $A$-module. Applying $D$ to this free resolution gives a levelwise free simplicial unstable $A$-module, so this is immediate from Theorem 4.20.

\section{Examples}

Corollary 4.17 reduces the analysis of the $E_{2}$-term of our spectral sequence to the computation of the derived functors of $D$. In this section we will apply results about these functors from the literature to describe the spectral sequence in two simple examples.

\subsection{Eilenberg-Mac Lane spectra}

The spectral sequence is clearly trivial for Eilenberg-Mac Lane spectra having the form $\Sigma^{k} \mathrm{HF}_{2}$. For a slightly less trivial example, consider the Eilenberg-Mac Lane spectra $\Sigma^{k} \mathrm{HZ}$ and $\Sigma^{k} \mathrm{HZ} / 2^{n}$, where $k$ must be positive for our convergence result to apply. The mod-2 cohomology of the spectrum $\mathrm{HZ}$, originally computed by Serre [29], is the $A$-module $A / \mathrm{Sq}^{1}$. Since $\mathrm{Sq}^{1} \mathrm{Sq}^{1}=0$, this has a simple free resolution, namely

$$
\cdots \rightarrow \Sigma^{2} A \stackrel{\cdot \mathrm{Sq}^{1}}{\longrightarrow} \Sigma A \stackrel{\cdot \mathrm{Sq}^{1}}{\longrightarrow} A .
$$

From this we see that, writing $F(n)=D\left(\Sigma^{n} A\right)$ for the free unstable $A$-module on a generator in degree $n$, the derived functors $\mathbb{L}_{*} D\left(\mathrm{H}^{*} \Sigma^{k} \mathrm{H} \mathbb{Z}\right)$ are given by the cohomology of the complex

$$
\cdots \rightarrow F(k+2) \stackrel{\cdot \mathrm{Sq}^{1}}{\longrightarrow} F(k+1) \stackrel{\cdot \mathrm{Sq}^{1}}{\longrightarrow} F(k) .
$$

But it is easy to see that this complex is exact for $k>0$, and so $\mathbb{L}_{*} D\left(\mathrm{H}^{*} \Sigma^{k} \mathrm{HZ}\right)$ is 0 for $*>0$. It follows that our spectral sequence has only a single column, and so collapses to give

$$
\mathrm{H}^{*}(K(\mathbb{Z}, k))=\mathrm{H}^{*}\left(\Omega^{\infty} \Sigma^{k} \mathrm{H} \mathbb{Z}\right) \cong U\left(F(k) / \mathrm{Sq}^{1}\right) .
$$

Similarly, the spectrum $\Sigma^{k} \mathrm{H} \mathbb{Z} / 2^{n}$ has cohomology $A / \mathrm{Sq}^{1} \oplus \Sigma A / \mathrm{Sq}^{1}$, so again $D$ has no derived functors and $\mathrm{H}^{*}\left(K\left(\mathbb{Z} / 2^{n}, k\right)\right) \cong U\left(F(k) / \mathrm{Sq}^{1}\right) \otimes U\left(F(k+1) / \mathrm{Sq}^{1}\right)$. Of course, these results agree with Serre's computations in [29]. 


\subsection{Suspension spectra}

In this subsection we consider the spectral sequence for infinite loop spaces of the form $\Omega^{\infty} \Sigma^{\infty} X$, where $X$ is a connected space. We will show, by a dimension-counting argument, that the spectral sequence collapses in this case.

The cohomology $\mathrm{H}^{*}\left(\Sigma^{\infty} X\right) \cong \widetilde{\mathrm{H}}^{*} X$ is an unstable $A$-module. Lannes and Zarati [19] computed the derived functors $\mathbb{L}_{*}(D)(M)$ for an unstable $A$-module $M$. An alternative computation (in the dual, homological, case), using a chain complex originally due to Singer [30], has been given by Kuhn and McCarty [18], and we will use their formulation of the result. Before stating this, we must introduce some notation:

Definition 5.1 Let $M$ be an $A$-module. Let $\mathcal{R}_{S} M$ be the quotient of the graded $\mathbb{F}_{2}$-vector space generated by symbols $Q^{I} x$ in degree $|x|+i_{1}+\cdots+i_{s}$, where $x \in M$ and $I=\left(i_{1}, \ldots, i_{s}\right)$, by the instability and Adém relations for the Dyer-Lashof algebra as well as linearity relations $\left(Q^{I}(x+y)=Q^{I} x+Q^{I} y\right)$. This becomes an $A$-module via the (dual) Nishida relation

$$
\mathrm{Sq}^{i} Q^{j} x=\sum_{k}\left(\begin{array}{c}
j-k \\
i-2 k
\end{array}\right) Q^{i+j-k} \mathrm{Sq}^{k} x .
$$

Let $d: \mathcal{R}_{S}(\Sigma M) \rightarrow \mathcal{R}_{s-1}(M)$ be defined by $d\left(Q^{I} \sigma x\right)=Q^{i_{1}, \ldots, i_{s-1}}\left(\mathrm{Sq}^{i_{s}+1} x\right)$; this is a map of $A$-modules. Writing $R_{S} M:=\Sigma \mathcal{R}_{S} \Sigma^{s-1}$ we can think of $d$ as a map $R_{S} M \rightarrow R_{S-1} M$.

The result, in the form given by [18, Theorems 4.22 and 4.34], is then:

Theorem 5.2 (Singer, Lannes and Zarati, Kuhn and McCarty) Let $M$ be an $A-$ module.

(i) The sequence

$$
\cdots \rightarrow R_{S} M \stackrel{d}{\rightarrow} R_{S-1} M \rightarrow \cdots \rightarrow R_{0} M
$$

is a chain complex, and $\mathrm{H}_{*}\left(R_{*} M\right)$ is naturally isomorphic to $\mathbb{L}_{*}(D)(M)$.

(ii) If $M$ is an unstable $A$-module, then the differential in $R_{*} M$ is zero and thus $\mathbb{L}_{*} D(M) \cong R_{*} M$.

(iii) If $M$ is an unstable $A$-module, then $\mathbb{L}_{s} D(M)$ is an $s$-fold suspension of an unstable module. 
By (iii), it follows that for $M$ unstable all the top squares in $\mathbb{L}_{s} D(M)$ are zero for $s>0$. By Corollary 4.17 we therefore have an isomorphism

$$
\mathbb{L}_{*}(U D)(M) \cong U(M)[0] \otimes \mathfrak{E}\left(\mathbb{L}_{*>0} D(M)\right) .
$$

Thus if $E$ is a spectrum such that $\mathrm{H}^{*} E$ is an unstable $A$-module, in the $E^{2}$-term of our spectral sequence for $\mathrm{H}^{*}\left(\Omega^{\infty} E\right)$ an element $v \in \mathrm{H}^{k} E$ gives:

- $\sigma Q^{I} \sigma^{s-1} v$ in degree $(-s, k+|I|+s)$, where $I=\left(i_{1}, \ldots, i_{s}\right)$ is an allowable sequence, ie $i_{t} \leq 2 i_{t+1}$, and $i_{1}>i_{2}+\cdots+i_{s}+|v|+s-1$ (for brevity we'll denote this element by $\left.\bar{Q}^{I} v\right)$;

- $\delta_{J} \bar{Q}^{I} v$ in degree $\left(-s-|J|, 2^{l}(k+|I|+s)\right)$, for $J$ an admissible sequence of length $l$.

The $E_{2}$-page of the spectral sequence is an exterior algebra on these generators.

Now suppose $X$ is a connected space of finite type; then $\mathrm{H}^{*} X \cong\left(\mathrm{H}_{*}(X)\right)^{\vee}$. In this case the spectral sequence for $\Sigma^{\infty} X$ converges by Theorem 2.1 . We wish to compare the $E_{2}$-page to the known cohomology $\mathrm{H}^{*}(Q X) \cong\left(\mathrm{H}_{*}(Q X)\right)^{\vee}$. Recall that the homology $\mathrm{H}_{*}(Q X)$ can be described in terms of the Dyer-Lashof operations $Q^{j}$ :

Theorem 5.3 (May [6]) If $X$ is a space, the homology $\mathrm{H}_{*}(Q X)$ is a polynomial algebra on generators $Q^{J} v$ where $v$ ranges over a basis of $\mathrm{H}_{*}(X)$, and $J=\left(j_{1}, \ldots, j_{s}\right)$ is an allowable sequence, meaning $j_{t} \leq 2 j_{t+1}$ for all $t$, and $j_{1}>j_{2}+\ldots+j_{s}+|v|$. The element $Q^{J} v$ is in degree $|v|+|J|$.

To see that the spectral sequence must collapse, it suffices to prove the following:

Proposition 5.4 There is a grading-preserving bijection between the exterior algebra generators $\bar{Q}^{I} x, \delta_{J} \bar{Q}^{I} x$ of the $E_{2}$-page and the Dyer-Lashof operations $Q^{K} x$, together with their powers $\left(Q^{K} x\right)^{2^{r}}$, for each $x$ in a basis for the reduced cohomology of $X$.

Proof Write $k:=|x|$. Observe that for any allowable sequence $I$ with

$$
i_{1}>i_{2}+\cdots+i_{s}+k+s-1,
$$

the total degree of $\bar{Q}^{I} x$ is the same as the degree of $Q^{I} x$. However, there are more nonzero Dyer-Lashof operations on $x$ than those given by these sequences: we are missing those where

$$
i_{2}+\cdots+i_{s}+k<i_{1} \leq i_{2}+\cdots+i_{s}+|x|+s-1 .
$$


To relate these to the $E_{2}$-term, we change the indexing of the Dyer-Lashof operations: If $J=\left(j_{1}, \ldots, j_{s}\right)$ is an allowable sequence, then for $Q^{J} v$ to be nonzero (and not a square) in $\mathrm{H}_{*}(Q X)$ we must have, for positive integers $l_{1}, \ldots, l_{s}$,

$$
j_{i}=k+j_{s}+j_{s-1}+\cdots+j_{i+1}+l_{i} .
$$

The allowability condition, expressed in terms of the $l_{i}$, says that $l_{i} \leq l_{i+1}$. Thus there exist nonnegative integers $a_{1}, \ldots, a_{s}$ (with $a_{1}>0$ ) such that $l_{i+1}=l_{i}+a_{i+1}$ (and $\left.l_{1}=a_{1}\right)$. In terms of the $a_{i}$ the element $Q^{J} x$ has degree

$$
2^{s} k+\sum_{j=1}^{s} \sum_{r=1}^{j} 2^{j-1} a_{r}=2^{s} k+\sum_{r=1}^{s}\left(\sum_{j=r}^{s} 2^{j-1}\right) a_{r}=2^{s} k+\sum_{r=1}^{s}\left(2^{s}-2^{r-1}\right) a_{r} .
$$

Let's write $q^{a_{1}, \ldots, a_{s}} x$ for the element $Q^{J} x$ with $J$ of this form. We also extend the notation by writing $q^{0, a_{1}, \ldots, a_{s}} x$ for $\left(q^{a_{1}, \ldots, a_{s}} x\right)^{2}$, etc.

Defining $\bar{q}^{a_{1}, \ldots, a_{s}} x$ similarly, we see that $\bar{q}^{a_{1}, \ldots, a_{s}} x$ and $q^{a_{1}+(s-1), a_{2}, \ldots, a_{s}} x$ have the same degree in $\mathrm{H}_{*}(Q X)$.

Now suppose $\delta_{I}$ is an admissible sequence of $\delta$-operations of length $l$. Then there exist nonnegative integers $r_{t}$ such that $i_{l}=r_{l} \geq 1$ and $i_{t}=2 i_{t+1}+r_{t}$ for $t<l$; in terms of the $r_{t}$ the admissibility criterion says that $r_{1}+\cdots+r_{l} \leq s$, and $|I|=\sum_{i}\left(2^{i}-1\right) r_{i}$. Then the total degree of $\delta_{I} \bar{q}^{a_{1}, \ldots, a_{s}} x$ is the same as the degree of $q^{K} x$, where

$$
K=\left(s-\sum r_{t}, r_{1}, r_{2}, \ldots, r_{l-1}, a_{1}+r_{l}-1, a_{2}, \ldots, a_{s}\right) .
$$

To see that this gives a bijection between the generators, we describe its inverse: For $q^{b_{1}, \ldots, b_{\sigma}} x$ in $H_{*}(Q X)$, let $L$ be the unique integer with $0 \leq L<\sigma$ such that

$$
b_{1}+\cdots+b_{L}+L<\sigma \leq b_{1}+\cdots+b_{L+1}+L+1 .
$$

Then we define

$$
\begin{aligned}
s & :=\sigma-L, \\
r_{L} & :=s-b_{1}-\cdots-b_{L}-L \quad \text { and } \quad r_{t}:=b_{t+1} \text { for } t=1, \ldots, L-1, \\
a_{1} & :=b_{L+1}-r_{L}+1 \quad \text { and } \quad a_{i}:=b_{L+i} \text { for } i=2, \ldots, s .
\end{aligned}
$$

Then

$$
\left(b_{1}, \ldots, b_{\sigma}\right)=\left(s-L-\sum_{t=1}^{l} r_{t}, r_{1}, \ldots, r_{L-1}, a_{1}+r_{L}-1, a_{2}, \ldots, a_{s}\right),
$$

so $q^{b_{1}, \ldots, b_{\sigma}}$ corresponds to $\delta_{I} \bar{q}^{a_{1}, \ldots, a_{s}} x$ where $I=\left(i_{1}, \ldots, i_{L}\right)$ is the admissible sequence determined by the $r_{t}$, ie with $i_{t}:=\sum_{j=t}^{L} 2^{j-t} r_{j}$. 
Corollary 5.5 For $X$ a connected space of finite type, the spectral sequence

$$
\mathbb{L}_{*}(U D)\left(\mathrm{H}^{*} X\right) \Rightarrow \mathrm{H}^{*}(Q X)
$$

collapses at the $E_{2}$-page.

\section{References}

[1] A K Bousfield, Operations on derived functors of nonadditive functors, unpublished manuscript, Brandeis University (1967)

[2] A K Bousfield, The localization of spectra with respect to homology, Topology 18 (1979) 257-281 MR

[3] A K Bousfield, On the homology spectral sequence of a cosimplicial space, Amer. J. Math. 109 (1987) 361-394 MR

[4] A K Bousfield, D M Kan, Homotopy limits, completions and localizations, Lecture Notes in Mathematics 304, Springer (1972) MR

[5] H Cartan, Puissances divisées, from "Séminaire Henri Cartan, 1954/1955 (Exposé 7)", volume 7, Secrétariat Mathématique, Paris (1955)

[6] F R Cohen, T J Lada, J P May, The homology of iterated loop spaces, Lecture Notes in Mathematics 533, Springer (1976) MR

[7] A Dold, Homology of symmetric products and other functors of complexes, Ann. of Math. 68 (1958) 54-80 MR

[8] A Dold, D Puppe, Homologie nicht-additiver Funktoren: Anwendungen, Ann. Inst. Fourier Grenoble 11 (1961) 201-312 MR

[9] W G Dwyer, Higher divided squares in second-quadrant spectral sequences, Trans. Amer. Math. Soc. 260 (1980) 437-447 MR

[10] W G Dwyer, Homotopy operations for simplicial commutative algebras, Trans. Amer. Math. Soc. 260 (1980) 421-435 MR

[11] PG Goerss, Unstable projectives and stable Ext: with applications, Proc. London Math. Soc. 53 (1986) 539-561 MR

[12] P G Goerss, On the André-Quillen cohomology of commutative $\mathbf{F}_{2}$-algebras, Astérisque 186, Société Mathématique de France, Paris (1990) MR

[13] P G Goerss, T J Lada, Relations among homotopy operations for simplicial commutative algebras, Proc. Amer. Math. Soc. 123 (1995) 2637-2641 MR

[14] A Grothendieck, Sur quelques points d'algèbre homologique, Tôhoku Math. J. 9 (1957) 119-221 MR

[15] P Hackney, Operations in the homology spectral sequence of a cosimplicial infinite loop space, J. Pure Appl. Algebra 217 (2013) 1350-1377 MR 
[16] S B Isaacson, Symmetric cubical sets, preprint (2009) arXiv (the published version at J. Pure Appl. Algebra 215 (2011) 1146-1173 does not include the relevant result)

[17] D M Kan, Functors involving c.s.s. complexes, Trans. Amer. Math. Soc. 87 (1958) 330-346 MR

[18] N Kuhn, J McCarty, The mod 2 homology of infinite loopspaces, Algebr. Geom. Topol. 13 (2013) 687-745 MR

[19] J Lannes, S Zarati, Sur les foncteurs dérivés de la déstabilisation, Math. Z. 194 (1987) 25-59 MR

[20] S Mac Lane, Categories for the working mathematician, 2nd edition, Graduate Texts in Mathematics 5, Springer (1998) MR

[21] H Miller, A spectral sequence for the homology of an infinite delooping, Pacific J. Math. 79 (1978) 139-155 MR

[22] H Miller, The Sullivan conjecture on maps from classifying spaces, Ann. of Math. 120 (1984) 39-87 MR

[23] H Miller, Correction to [22], Ann. of Math. 121 (1985) 605-609 MR

[24] G M L Powell, On the derived functors of destabilization at odd primes, Acta Math. Vietnam. 39 (2014) 205-236 MR

[25] D G Quillen, Homotopical algebra, Lecture Notes in Mathematics 43, Springer (1967) MR

[26] C Rezk, Every homotopy theory of simplicial algebras admits a proper model, Topology Appl. 119 (2002) 65-94 MR

[27] S Schwede, B E Shipley, Algebras and modules in monoidal model categories, Proc. London Math. Soc. 80 (2000) 491-511 MR

[28] S Schwede, B Shipley, Equivalences of monoidal model categories, Algebr. Geom. Topol. 3 (2003) 287-334 MR

[29] J-P Serre, Cohomologie modulo 2 des complexes d'Eilenberg-MacLane, Comment. Math. Helv. 27 (1953) 198-232 MR

[30] W M Singer, Iterated loop functors and the homology of the Steenrod algebra, II: A chain complex for $\Omega_{s}^{k} M$, J. Pure Appl. Algebra 16 (1980) 85-97 MR

[31] W M Singer, A new chain complex for the homology of the Steenrod algebra, Math. Proc. Cambridge Philos. Soc. 90 (1981) 279-292 MR

[32] J M Turner, On simplicial commutative algebras with vanishing André-Quillen homology, Invent. Math. 142 (2000) 547-558 MR 
Department of Mathematical Sciences, University of Copenhagen

Universitetsparken 5, DK-2100 Copenhagen, Denmark

Department of Mathematics, Massachusetts Institute of Technology

Building 2, Room 106, 77 Massachusetts Avenue, Cambridge, MA 02139-4307, United States haugseng@math.ku.dk, hrm@math.mit.edu

http://sites.google.com/site/runehaugseng, http://math.mit.edu/ hrm

Received: 13 August $2015 \quad$ Revised: 23 February 2016 
